\title{
Probabilistic Learning Curve and Real Options Approach to the Valuation of Cross-Training with Product Life Cycle
}

\author{
by \\ Thanasiri Muttulingam \\ B.Eng.(Computer System Engineering) \\ Carleton University, \\ Ottawa, ON, November 2005 \\ M.A.Sc.(Computer and Electrical Engineering) \\ Carleton University, \\ Ottawa, ON, November 2007
}

A Thesis

presented to Ryerson University

in partial fulfillment of the

requirements for the degree of

Master in Applied Science

in the Program of

Mechanical Engineering

Toronto, Ontario, Canada, 2012

(C) Thanasiri Muttulingam 2012 


\section{Author's Declaration}

I hereby declare that I am the sole author of this project.

I authorize Ryerson University to lend this project to other institutions or individuals for the purpose of scholarly research.

Thanasiri Muttulingam

I further authorize Ryerson University to reproduce this project by photocopying or by other means, in total or in part, at the request of other institutions or individuals for the purpose of scholarly research.

Thanasiri Muttulingam 


\section{Borrower's Page}

Ryerson University requires the signatures of all persons using or photocopying this project. Please sign below, and give address and date. 


\title{
Probabilistic Learning Curve and Real Options Approach to the Valuation of Cross-Training with Product Life Cycle
}

\author{
Thanasiri Muttulingam
}

Master in Applied Science, Mechanical Engineering, 2012 Ryerson University

\begin{abstract}
Learning and forgetting are two important characteristics in manufacturing environments where workers are cross-trained to increase their flexibility of adapting to different tasks. Cross-training is introduced by industries so that one worker can work on multiple stations. This thesis develops two models: (i) a probabilistic learning curve approach to the production lot size problem to determine the economic manufactured quantity (EMQ); (ii) a real options approach to the valuation of cross-training with product life cycle. Different workers perceive the complexity of a certain task differently and each worker will have his/her learning curve with its individual characteristics. So, it is more realistic to assume that the learning curve characteristics are random variables with given probability density functions. Furthermore, for the second model, the demand of the product follows three-regime product life cycle. Each regime is modeled by a geometric Brownian motion. The net present value (NPV) is calculated using the real options. The results show that there is a significant change in the NPV compare to standard model with simplified assumptions.
\end{abstract}




\section{Acknowledgements}

I am deeply indebted to my project supervisors, Dr. Mohamed W. M. Ismail and Dr.

Mohamed Y. Jabar, for their valued and generous guidance and encouragement. Their patience has played fundamental role in the completion of this project. I am very grateful for giving me this opportunity to work under their supervision.

I thank Social Sciences and Humanities Research Council (SSHRC) of Canada for supporting my research.

At the same time, I appreciate the generous support given to me by my husband, parents, and Jeyanthy machal. 


\section{Table of Contents}

Author's Declaration $\quad$ ii

Borrower's Page iii

Abstract $\quad$ iv

Acknowledgement $\quad$ v

Table of Contents $\quad$ vi

List of Tables $\quad$ vii

List of Figures $\quad$ viii

Nomenclature $\quad x$

1 Introduction 1

2 Literature Review 5

2.1 Probabilistic learning curve and the effect of learning and forgetting on

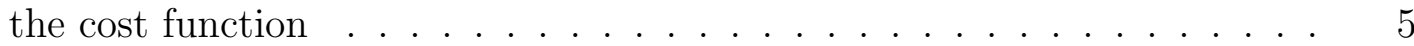

2.2 Real options for cross-training with learning and product life cycle . . . 7

2.3 Serial production system release policy . . . . . . . . . . . . . . . 11 
3.1 The deterministic learning curve model . . . . . . . . . . . . . . . 18

3.1.1 The infinite planning horizon model . . . . . . . . . . . . . . . . 18

3.1.2 The finite planning horizon model . . . . . . . . . . . . . . . . . . 19

3.2 The probabilistic learning curve model . . . . . . . . . . . . . . . 20

3.2.1 The infinite planning horizon model . . . . . . . . . . . . . . . 22

3.2.2 The finite planning horizon model . . . . . . . . . . . . . . . . . 24

3.3 Numerical example and analysis . . . . . . . . . . . . . . . 25

4 Real Options for Cross-Training Model 35

4.1 Optimization models . . . . . . . . . . . . . . . 38

4.1.1 Optimization model with cross-training . . . . . . . . . . . . 39

4.1.2 Optimization model without cross-training . . . . . . . . . . . 41

4.2 Dynamic programming . . . . . . . . . . . . . . . . 42

4.3 Numerical analysis . . . . . . . . . . . . . . . . . . . . . . . . 44

5 Conclusion $\quad 55$

$\begin{array}{ll}\text { Appendix } & 58\end{array}$

$\begin{array}{ll}\text { References } & 59\end{array}$ 


\section{List of Tables}

3.1 Optimum quantity and estimated optimum quantity produced for 12 cycles for $t_{B}=300$ and 500 days, respectively . . . . . . . . . . . . 28

3.2 Optimum quantity and estimated optimum quantity produced for 12 cycles for $t_{B}=1000$ and 3000 days, respectively . . . . . . . . . . . .

3.3 Optimum quantity and estimated optimum quantity produced for 12 cycles for $t_{B}=6000$ and 12000 days, respectively . . . . . . . . . . 30

4.1 Annual risk adjusted mean and volatility for each regime of product life

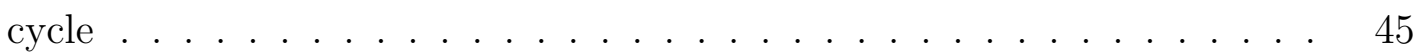

4.2 Holding costs involved in each station . . . . . . . . . . . . 45

4.3 Learning slope of each worker at each station . . . . . . . . . . . . . 45

4.4 Time to produce first unit for each worker at each station . . . . . . . . 45

4.5 Labor cost for each worker . . . . . . . . . . . . . . . 46

4.6 Cross-training cost for each worker f . . . . . . . . . . . . 46 


\section{List of Figures}

3.1 Learning curve . . . . . . . . . . . . . . . . . . . . . . . . 14

3.2 Forgetting curve . . . . . . . . . . . . . . . . . 15

3.3 Learn-forget curve . . . . . . . . . . . . . . . . . . . . . . 16

3.4 Inventory level affected by learning . . . . . . . . . . . . . . . 18

3.5 Effect on the output when $T_{1 b}$ changes . . . . . . . . . . . . . 31

3.6 Effect on the output when $T_{1 a}$ changes $\ldots \ldots \ldots \ldots$

3.7 Effect on the output when $l_{b}$ changes . . . . . . . . . . . . . . 33

3.8 Effect on the output when $l_{a}$ changes $\ldots \ldots \ldots \ldots$

4.1 Heptanomial lattice . . . . . . . . . . . . . . . . . . . 37

4.2 Cross-training status for three periods of binomial lattice . . . . . . . . . 47

4.3 Cross-training status for three periods of heptanomial lattice . . . . . . . 48

4.4 Change in profit vs. volatility of growth regime $\left(\sigma_{g}\right) \ldots \ldots . \ldots . \ldots$

4.5 Change in profit vs. volatility of maturity regime $\left(\sigma_{m}\right) \ldots \ldots$

4.6 Change in profit vs. volatility of decay regime $\left(\sigma_{d}\right) \ldots \ldots \ldots$. . . . . 51

4.7 Change in profit vs. annual risk adjusted mean $\left(\mu_{g}\right) \ldots \ldots \ldots 2$

4.8 Change in profit vs. years of lifecycle . . . . . . . . . . . . . . 52

4.9 Change in profit vs. number of stations cross-trained . . . . . . . . 53

4.10 Change in profit vs. number of workers cross-trained . . . . . . . . . 54 


\section{Nomenclature}

y Cumulative production count

$x \quad$ Number of output units that would have been if no interruption occurred

$r \quad$ Consumption rate

$q_{i} \quad$ Units produced in each cycle $i$

$\alpha_{i} \quad$ Number of units accumulated at the beginning of the cycle $i$

$T_{y} \quad$ Time required to produce the $y^{\text {th }}$ unit

$T_{1} \quad$ Time required to produce the first unit

$T_{x} \quad$ Time for the $x^{\text {th }}$ unit of lost experience

$t_{B} \quad$ Break time over which the total forgetting occurs

$t_{p i} \quad$ Production time for cycle $i$

$t_{b i} \quad$ Break time for cycle $i$

$t_{c i} \quad$ Total cycle time for cycle $i$

$t\left(q_{i}\right) \quad$ Total time to produce $q_{i}$ units

$T_{q_{i}+\alpha_{i}} \quad$ Time required to produce the $q_{i}^{t h}$ unit in cycle $i$ with the beginning units $\alpha_{i}$

$t^{s}\left(q_{i}\right) \quad$ Total time to produce $q_{i}$ units in stochastic learning curve equation

$T_{1 a} \quad$ Lower limit for the time required to produce first unit, $T_{1}$

$T_{1 b} \quad$ Upper limit for the time required to produce first unit, $T_{1}$

$f\left(T_{1}\right) \quad$ Uniform distribution function for $T_{1}$

$\tau_{j k} \quad$ Time to produce the first item at station $j$ by worker $k$

$l \quad$ Learning curve slope

$l_{a} \quad$ Lower limit for learning curve slope, $l$

$l_{b} \quad$ Upper limit for learning curve slope, $l$

$f(l) \quad$ Uniform distribution function for learning curve slope, $l$

$l_{j k} \quad$ Learning curve slop of worker $k$ at station $j$

f $\quad$ Forgetting curve slope

$C_{i} \quad$ Minimum value of the ratio of the break time duration to the production time in cycle $i$ 
$R_{i} \quad$ Number of units that would have been produced if no interruption occurred in cycle $i$

$Z_{i} \quad$ Maximum inventory level in cycle $i$

$I_{j t} \quad$ Inventory at station $j$ at the end of period $t$

B The matrix represents all possible inventory levels

I A raw vector in matrix $\mathbf{B}$

$k \quad$ Setup cost

$h_{j} \quad$ Inventory carrying cost per unit per unit time per station $j$

$d_{m} \quad$ Material cost

$\gamma \quad$ Labor cost

$C_{j k} \quad$ Cross-training cost at station $j$ for worker k

$U \quad$ Unit cost per item (including raw material cost and setup cost)

$b_{j k} \quad$ Labor cost per hour of worker $k$ at station $j$

$N \quad$ Number of lots

$N^{*} \quad$ Optimum number of lots

$N_{L} \quad$ Lower limit of optimum number of lots

$N_{U} \quad$ Upper limit of optimum number of lots

$g \quad$ Growth regime

$m \quad$ Maturity regime

$d \quad$ Decay regime

$z \quad$ Level of the underlying variable the lattice

$\pi_{g, u} \quad$ Branch probability of the growth regime of the upward branch

$\pi_{g, m} \quad$ Branch probability of the growth regime of the middle branch

$\pi_{g, d} \quad$ Branch probability of the growth regime of the downward branch

$\pi_{m, u} \quad$ Branch probability of the maturity regime of the upward branch

$\pi_{m, m} \quad$ Branch probability of the maturity regime of the middle branch

$\pi_{m, d} \quad$ Branch probability of the maturity regime of the downward branch

$\pi_{d, u} \quad$ Branch probability of the decay regime of the upward branch 
$\pi_{d, m} \quad$ Branch probability of the decay regime of the middle branch

$\pi_{d, d} \quad$ Branch probability of the maturity regime of the downward branch

$\phi_{w} \quad$ Continuously compound rate of return or step size of the regime $w$

$\mu_{w} \quad$ Brownian motion drift parameter of the regime $w$ (mean demand per unit time)

$\sigma_{w} \quad$ Volatility of demand per unit time of the regime $w$

$\Delta t \quad$ Interval between two layers of the lattice

$p(t) \quad$ Probability of switching from growth regime to maturity regime in period $t$

$g(t) \quad$ Probability of switching from maturity regime to decay regime in period $t$

$\beta_{t} \quad$ Number of hours in a period $t$

$p \quad$ Price per item

$D_{t} \quad$ Demand at period $t$

$Q_{j t} \quad$ Production quantity at station $j$ during the period $t$

$X_{j k t} \quad$ Fraction of the time worker $k$ works at station $j$ in period $t$

$S_{j k t} \quad$ Cross-training status of worker $k$ to station $j$ in the period $t$ 


\section{Chapter 1}

\section{Introduction}

Many companies today try to manufacture products with the highest possible quality and to offer them in the right quantities at competitive prices so as to gain larger market share than their competitors. One of the way to achieve by cross-train the workers to work on multiple stations and considering learning in production. Research on the effects of learning and forgetting (its opposite phenomenon) on production planning and control policies is well documented in the literature (e.g., Jaber 2006). Learning and forgetting are two characteristics of workers in manufacturing (or other industries), especially, when workers alternate between different tasks to increases their flexibility. A policy that requires them to be cross-trained, where workers' learning is usually interrupted by breaks, or by a new task. This thesis will: (i) develop a probabilistic learning curve and then investigate the effects of learning and forgetting on the cost function for an inventory system; and (ii) introduce the concept of real options for the valuation of cross-training with learning and product life cycle.

On an assembly line, workers perform tasks repetitively. Repetition helps workers learn their job faster with time, which accelerates the production rate. Production interruption and cross-training impedes the performance of workers as a result of forgetting. When workers commence their work after an interruption, or after being away from a 
task, performing another one, for some time, or when a new product is introduced into the production line they need some time to regain their performance. This means cost to the company and loss of productivity. Surely, such costs cannot be avoided, but certainly could be minimized. This will be the focus of the thesis.

Unlike the models in the literature, this thesis makes a realistic assumption that the learning curve parameters, which are the initial processing time and the learning exponent, are not deterministic. These parameters are those of a learning curve that captures the learning of a population of workers (Nembhard and Uzumeri 2000). Practically, different workers perceive the complexity of a certain task differently. This suggests that each worker will have his/her learning curve with its individual characteristics. So, it is more realistic to assume that the learning curve characteristics are random variables with given probability density functions. This surely gives more accurate results than the aggregation of workers' learning curves. To demonstrate the importance of assuming a stochastic rather than a deterministic curve, and prior to addressing the cross-training problem, the model of Jaber and Bonney (1998) is modified to account for random learning curve parameters. The modified model and the deterministic one (Jaber and Bonney 1998) are compared and discussed.

The thesis also focuses on real options for a cross-training model. There are three cross-training schemes (Marks et al. 2002): (1) positional clarification where workers learn from verbal instructions conveyed by more experienced team members; (2) positional modeling where workers learn from verbal discussions and observations of the work of the other team members; and (3) positional rotation where workers learn from hands on approach. In this thesis, the positional rotation scheme is adopted as it is used by many companies.

Cross-training is useful to balance capacity (Bokhorst et al. 2004 and Iravani et al. 2005). It equally distributes the workload amongst workers at different workstations and improves the utilization and throughput of the production line. Cross-training also im- 
proves productivity without increasing the work-in-process where idle workers are moved to busy workstations to reduce bottle-neck situations. There are three questions to answer before a worker is assigned to a job: (1) what is his/her flexibility level (i.e., how many tasks he/she has been cross-trained to perform)?; (2) what levels of skill he/she has for the cross-trained tasks?; and (3) to which tasks a worker should be assigned to and for how long?. To answer these questions in this thesis, the following assumptions are made: (1) units are produced proportionally with cross-training; (2) each worker has to complete the assigned job before the beginning of the next shift; (3) each worker is fully trained for a job or not at all; and (4) cross-training assignments are dynamic.

Cross-training of workers has several benefits (Ebeling and Lee 1994): (1) improves meeting due dates; (2) increases workers' knowledge and skill levels about products and processes; (3) reduces overtime costs due to absenteeism; (4) smoothes the transition phase between products; (5) improves product and process quality; and (6) increases throughput. (e.g., Park and Bobrowski 1989 and Wisner and Siferd 1995). However, these benefits come at the cost of training and retraining of workers. Cross-training also has conflicting objectives. There will be additional cost for labor lost during crosstraining. Management would like to see workers' idle times reduced, which increases the workers' workload.

This thesis investigates cross-training in a production situation. The demand is uncertain and it follows three-regime product life cycle: growth regime, maturity regime and decay regime. Each regime is modeled by a geometric Brownian motion. The first regime of the product life cycle is the growth regime. Afterwards, it switches stochastically to maturity regime and then to decay regime. The growth regime is represented with increasing demand, the maturity regime is represented with stabilized demand and the decay regime is represented with decreasing demand. To represent these regimes on the lattice, a heptanomial lattice is used, which has seven branches. A lattice is used to find the fair value of a an option. The lattice model divides the time between now 
and the option's expiration into $\mathrm{N}$ discrete periods. The option value is calculated with different level of inventories. Then the optimal present value is calculated using optimization models with non-linear programming. One optimization model calculates the present value without cross-training and another model calculates the present value with cross-training. The real options is used to find the highest profit. The assumption the thesis makes for the production process is that the production floor has workstations, primary workers, and secondary workers. A Primary worker works on his or her primary workstation. A Secondary worker works on a different workstation other than his or her primary workstation. The results show that there is a significant change in the present value, which is calculated with the cross-training, learning, product life cycle and stochastic demand, compared to standard model with simplified assumptions.

The reminder of this study is organized as follows. Chapter 2 has background information. Chapter 3 is to elaborate the probabilistic learning curve model with a numerical example and the discussion. Chapter 4 describes the dynamic programming and the real options models with a numerical example and the results. Chapter 5 concludes the study of the findings and the future work. 


\section{Chapter 2}

\section{Literature Review}

This chapter provides a brief introduction about the basic concept of probabilistic learning curve, learning and forgetting, cross-training, and real options. It includes a briefing of the problems and benefits as analyzed by multiple authors.

\subsection{Probabilistic learning curve and the effect of learn- ing and forgetting on the cost function}

Many psychologists experiment on individual learning ability (Mazur and Hastie 1978). There are many studies and models present in the literature: 1) how one organization keeps hold of knowledge over time (Epple et al. 1991), 2) how learning is related to manufacturing cost (Yelle 1979), 3) how learning is related to process time (Adler and Nanda 1974, Axsater and Elmaghraby 1981, Smunt 1987, and Sule 1981), 4) how learning is related to setup time (Karwan et al. 1988 and Pratsini et al. 1993), 5) how learning is related to product veracity and process complexity (McCreery and Krajewski 1993) and 6) how learning is related to product and process quality (Jaber and Guiffrida 2004 and Jaber and Guiffrida 2008).

This research is related to Jaber and Bonney (1998), Mazzola and McCardle (1997), 
and Wright (1936), which use the traditional learning curve model. Learning curve theory can capture how fast workers can learn. The conventional learning curve is power form and it is in labor hours per unit versus cumulative number of units (Wright 1936). The traditional learning curve may include employee, organizational system and outside factors.

The learning and forgetting model of Shafer et al. (2001) was formed using the following parameters: productivity rate for cumulative work, initial expertise of workers units of cumulative work, steady- state productivity rate after all the learning has been completed, learning rate from cumulative work and initial expertise require to reach half of steady-state productivity rate, how recent the workers learn, and forgetting rate from how recent the workers learn. Their results suggest that reducing the forgetting rate is not important in a fast learning environment. Benefits of smaller size of worker pool increase as the learning rate and the length of task tenures decreases. Increasing the length of task tenures is not increasing productivity of the system. Increasing the length of task tenures is more beneficial when there is large worker pool but not much effect on smaller worker pool. Increasing the learning rate moderate the negative effect of increasing the size of worker pool and the forgetting rate (Yelle 1979 and Shafer et al. 2001).

Although the work in this thesis is relevant to that of Nembhard and Shafer (2008), it differs from it. Our model is based on a well founded industrial learning curve, where as Nembhard and Shafer (2008) is not. They use a learning curve from psychology (Mazur and Hastie 1978) that is based on the initial expertise, steady-state productivity, rate of learning and rate of forgetting of heterogeneity in workers, where higher heterogeneity in workers corresponds to higher productivity. From analytical and simulation results, increasing the variance in steady state of worker productivity does not change the productivity level. However, increasing the variance of the initial processing time decreases the productivity level. Increasing the variance in the learning rate of workers or the forgetting rate increases the overall output. From simulation results, heterogene- 
ity in conjunction with learning rate has the most impact on the system's productivity. Increasing the initial processing time decreases the learning rate.

Nembhard (2001) investigates the heuristic policy for choosing workers for specific tasks based on the learning rate of individual workers. It increases overall productivity. A heterogeneous worker pool is more beneficial than a homogenous worker pool to increase the productivity. Short production cycles give more improvements. Gradual learning gives greater improvement instead of rapid learning. The heuristic policy will have more impact if the workers need more practice, and workers have less variance and mean than previous experience.

Models of Mazzola and McCardle (1997) and Salameh et al. (1993) are relevant to the model, which is developed in this thesis and explores the probabilistic learning curve. When the deterministic learning curve model was used, it gives more optimal production quantity than myopic production. But, Mazzola and McCardle (1997) and Salameh et al. (1993) showed that this is not always true if the probabilistic learning curve model is used.

\subsection{Real options for cross-training with learning and product life cycle}

Many researchers have analyzed the cost benefits of cross-training (Ebeling and Lee 1994, Nembhard et al. 2005, Bollen 1999, Hopp and VanOyen 2004, Katok et al. 1998, Shaw and Wagelmans 1998, VanMieghem 1995, and Nembhard et al. 2002). Ebeling and Lee (1994) argued that the optimal solution is to train fewer workers for more extensive jobs; it is costly and longer time to payoff. High attendance workers should train for a greater number of jobs if the jobs contribute greatly to overtime cost. When the variation of the attendance is less, the cross-training is evenly spread among the employees. Crosstraining is sensitive to the values of the overtime cost. The greater the overtime costs, 
the faster the payoff from the cross-training. Katok et al. (1998) discussed about the coefficient modification heuristic with cost balancing and setup reduction. VanMieghem (1995) and Nembhard et al. (2002) analyzed how to weigh the costs of cross-training. Shaw and Wagelmans (1998) argued about a dynamic programming algorithm problem with a general cost structure.

However, many researchers have analyzed the production flexibility (Bessant and Haywood 1988, Sethi and Sethi 1990, Upton 1994, Upton 1995, VanMieghem 1995, Brusco and Johns 1998, Choi and Kim 1998, Campbell 1999, Nembhard et al. 2002, Slomp and Molleman 2002, Marks et al. 2002, Jaber et al. 2003, Hopp et al. 2004, and Zamiska et al. 2007). For example, Slomp and Molleman (2002) concentrated on the development of team member flexibility by cross-training. Four cross-training policies were considered: critical task policy, worker flexibility policy, random policy and bottleneck redundancy policy. Slomp and Molleman (2002) identified that cross-training each worker to have equal number of skills is better with homogeneous workers. Bessant and Haywood (1988) argued that the flexibility is an organizational property rather than a technical one. Marks et al. (2002) examined the effects of the cross-training on shared knowledge structure as an indicator of cross-training effectiveness. Hopp et al. (2004) identified that cross-training the bottleneck workers can produce increases in throughput when the line is short and nearly balanced, variability of non-bottleneck stations is high and variability of the bottleneck is low. Campbell (1999) evaluated the benefits of cross-training and cross-utilization using a mathematical model and experiments. The mathematical model is called the allocation model and it is used to allocate cross-trained workers. The objective function for this model maximizes the sum of the utility associated with the assignment of workers to the department. If the capacity of workers is given as zero or one, the problem can be solved optimally. However, if it is given in the fraction, the value of cross-utilization is evaluated based on heuristic solution. The key factors that affect the cross-utilization are demand variation, cross-training breadth, 
and cross-training depth. The benefits of cross-utilization can be substantial with small degree of cross-training. After a certain value, the additional cross-training capture little additional value. The preferred value depends on demand variability. Upton (1994) and Upton (1995) described the dynamics that create the flexibility. VanMieghem (1995) and Nembhard et al. (2002) analyzed about the flexibility against the resulting performance benefits and profits. Sethi and Sethi (1990) and Choi and Kim (1998) examined the various forms of manufacturing flexibility with implications for cross-training.

Moreover, some researchers have examined the benefits in reducing the average number of customers in a queue (Iravani et al. 2007 and Iravani et al. 2005). Iravani et al. (2007) argued that cross-training allows customer service representative to dynamically relocate to answer calls from different services. It also reduces lack of calls for one customer service representative and more calls for another representative even in non-peak times. Iravani et al. (2005) developed a method to find an index for all the alternative structures to find what structure has robust and better performance without precise information on demand or capacity. So, the capacity structure should not tie to a specific demand or capacity pattern because otherwise it would reduce robustness of the system. System structure is a means for buffering against variability. The variability of demand can be met by either increasing the capacity or increasing the flexibility of available capacity such as using production sources with multiple capacities. In production sources with multiple capacities, the capacity can be used against variability by increasing capacity or shifting capacity among sources. The ability of shifting capacity as the result of capacity patterns of sources called system structure.

Furthermore, some researchers used real options to value cross-training policies. Nembhard et al. (2005) and Yang (2007) used real options framework to model and value cross-training policies. There are four factors that affects this model: 1) task heterogeneity, 2) worker heterogeneity, 3) labor dynamic (worker on leave), and 4) product dynamic (product change). Nembhard et al. (2005) and Yang (2007) considered net present value 
based on traditional discounted cash flow technique for the production system with specialized workers and cross-trained workers. Two binomial lattices are used for underlying production value and options valuation. If the system is already near optimal, the real options approach is not better than traditional discounted cash flow.

Brusco and Johns (1998), Hung and Chien (2000), and Katok et al. (1998) analyzed the workforce staffing assignment in terms of cost, productivity, policy level and multiple work activity categories. They recent integer linear programming mode with the objective to minimize workforce staffing cost. Cross-training structure, which is defined as a policy, contains number of work activity categories, the level of productivity and the framework for deciding what skill classes are trained for what work activity categories. They found that for all the cross-training structures with $100 \%$ productivity in secondary skill class are able to find the minimum cost for all the labor requirement patterns. Considerable benefits can be realized even in a limited use of cross-training. Even cross-training structures with $50 \%$ productivity in secondary skill class are able to find $86.9 \%$ of the cost saving of $100 \%$ productivity.

Yang (2007) concentrated on the choice between equal and unequal number of skills per worker. The results provide the right number of cross-trained workers, additional skills per cross-trained worker, and additional machines to realize the benefits of cross-training. The right cross-training policies were chose in different environments. The cross-training policies are compared by different efficiency losses, labor utilization, and variability in processing times, and worker absenteeism. The gap between benefits of cross-training and no cross-training decreases as efficiency loss increases. This gap increases as the variability in processing times increases. A positive efficiency loss implies that a worker increasingly losing his or her power to learn a new skill. It is better to cross-train workers to fewer works. 


\subsection{Serial production system release policy}

Production control can be identified with two systems: push and pull systems. The production is scheduled and the material is pushed into the production line is called push system. The completion of a product at the end of the production line triggers the start of next product assembly process is called pull system. One alternative of a pull system is the CONstant Work in Process (CONWIP) system. CONWIP is a mixture of a push-pull system. Many researchers have analyzed the CONWIP system: Geraghty and Heavey (2004), Marek et al. (2001), Spearman et al. (1990), Helber et al. (2011), Gaury et al. (2000), Ryan et al. (2000), Herer and Masin (1997), and Gstettner and Kuhn (1996).

CONWIP uses only one set of system cards to manage system WIP. CONWIP uses cards to control the number of WIPs. For example, no part is allowed to enter the system without a card. After a finished part is completed at the last workstation, a card is passed to the first workstation and a new part is pushed into the sequential process route (Marek et al. 2001).

A push system is where the jobs are scheduled and pull system is where the job to enter the system is triggered by the completion of another job. Pull system has the shorter flow time and reduced inventory level. Flow time or cycle time is the time between the job release and its completion. A job will not start until a place is vacated in the system. Enough jobs placed in the line so the bottleneck station is rarely idle. But the jobs do not have to wait long. It will give maximum throughput without excessive flow time or WIP (Helber et al. 2011).

The disadvantage of the CONWIP is that it does not control the inventory level individually inside the system. If one workstation breaks down, the inventory will pile up (Gaury et al. 2000). The objective of the Kanban is to decrease the WIP and of the push system is to increase the throughput (Amin and Altiok 1997). Kanban means marker or card in Japanese. To control WIP between workstations, Kanban uses card 
sets. Total WIP is equal to the summation of the number of cards in each card set. If raw material is available and the material has a card, then only the production occurs at a workstation. When the material receives card authorization to move, then only it is pulled through the system. Kanban system pulls work between every pair of workstations (Marek et al. 2001). In our case, we need to have a constant inventory level at each station. So, Kanban policy is chosen over CONWIP policy to work in this model. If CONWIP policy is selected, the finished inventory at each station cannot be maintained at a certain level.

In this study, the conventional learning curve will be analyzed (Jaber and Bonney 1998, Jaber and Bonney 1996, Jaber and Bonney 1997, Jaber and Sikstrom 2004, Mazzola and McCardle 1997, Wright 1936, and Salameh et al. 1993). Normally the learning slope and time required to produce the first unit are constant. However, in this study, we are going to modify the learning curve equation to have probabilistic learning slope and time required to produce the first unit. All the workers do not have the same learning rate or does not finish the work in same time frame. When we give probabilistic learning rate and time to do the units, it makes the process more realistic. There will be a significant difference when we use probabilistic parameters to model the learning curve instead of deterministic parameters. A numerical example using uniform distribution for both learning slope and the time to produce first unit is presented in the application of learning and forgetting to determine EMQ. Furthermore, we will analysis cross-training and real options. The demand of the product is uncertain and it follows three-regime product life cycle: growth, maturity, and decay. Each regime is modeled as geometric Brownian motion. The profit is calculated using non-linear program and the constraints include the conditions for learning with or without cross-training. The real options is used to choose the highest profit. The option value is calculated with different inventory levels. A numerical example is analyzed and the results show that there is a significant change in the profit compare to standard model with simplified assumptions. 


\section{Chapter 3}

\section{Probabilistic Learning Curve Model}

In this chapter, a probabilistic version of the Wright (1936) learning curve is introduced. This model can be used to investigate almost all learning and forgetting models that are available in the literature. Some of the models, which are relevant to the thesis, that assume learning to follow the (deterministic) Wright's learning curve are those of Salameh et al. (1993), Jaber and Bonney (1998), Jaber and Bonney (1996), Jaber and Bonney (1997), Jaber and Sikstrom (2004), and Jaber and Guiffrida (2004). In this chapter, the developed (probabilistic) model will be investigated in a lot sizing problem setting by revisiting the work of Jaber and Bonney (1998), who assumed learning to follow Wright's learning curve, whose parameters are deterministic. Practically, different workers perceive the complexity of a certain task differently. This suggests that each worker will have his/her learning curve with its individual parameters. So, it is more realistic to assume that the learning curve parameters are random variables with given probability density functions. This surely gives more accurate results than the aggregation of workers' learning curves. To demonstrate the importance of assuming a stochastic rather than a deterministic curve, the model of Jaber and Bonney (1998) is modified to account for random learning curve parameters.

The conventional learning curve phenomenon described by Wright (1936) has the 
labor hours per unit decrease when cumulative units increase as sown in Figure 3.1. The equation for the learning curve is as follows.

$$
T_{y}=T_{1} y^{-l}
$$

where $y$ is the cumulative production count, $T_{y}$ is the time required to produce the $y^{\text {th }}$ unit, $l$ is the learning curve slope and $T_{1}$ is the time required to produce the first unit. The parameters of the learning curve in Equation (3.1) are deterministic. For a population of workers, the learning curve in Equation (3.1) can be an aggregate learning curve that is averaging the output of each worker for each repetition and then fitting the learning curve to the aggregated data. Workers learn differently suggesting that each one has his/her own $T 1$ and $l$ values. As worker's learning styles vary and are independent from other learners, then it is reasonable to assume that the $T 1$ and $l$ values are random variables where each follows a probability density function. This assumption will be adopted in this chapter and throughout the thesis.

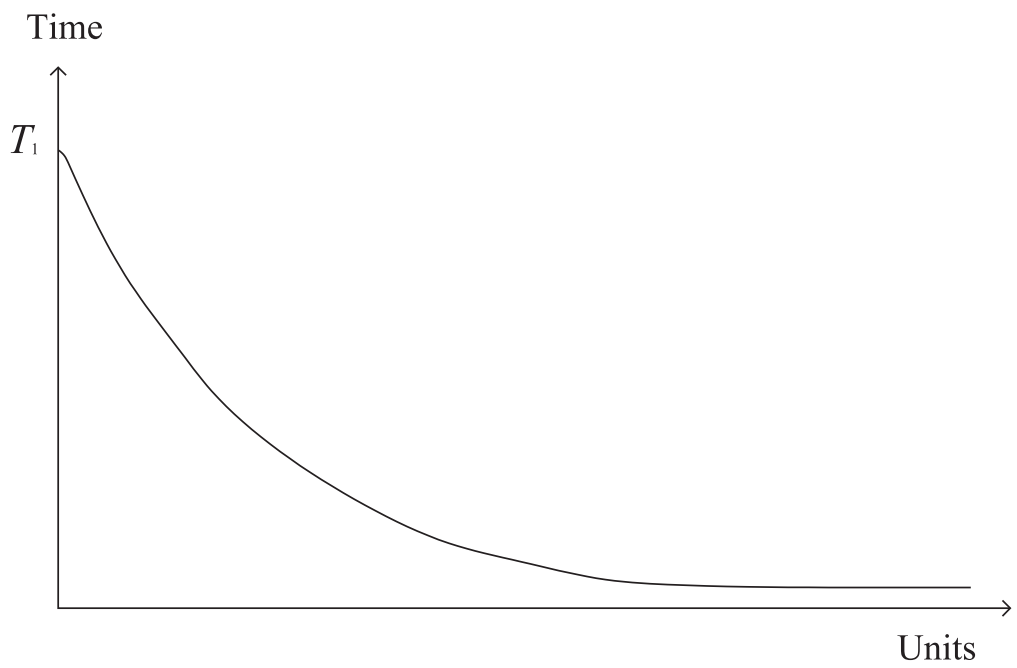

Figure 3.1: Learning curve

Wherever an interruption occurs in the learning process forgetting takes place (e.g., Jaber and Bonney 1998, Jaber and Bonney 1996, Jaber and Bonney 1997, and Jaber and 
Guiffrida 2004). The forgetting theory assumes that the time required to produce the first unit after an interruption (break) is dependent on the length of the break and the time it took to produce the last unit of the last cycle. The forgetting curve equation is as follows (Globerson et al. 1989 and Jaber and Bonney 1996).

$$
T_{x}=\hat{T}_{1} x^{f}
$$

where $x$ is the number of output units that would have been if no interruption occurred, $T_{x}$ is the time for the $x^{t h}$ unit of lost experience, $f$ is the forgetting slope and $\hat{T}_{1}$ is the time required for the first unit of forgetting exponent. Figure3.2 shows behavior of the forgetting curve Equation (3.2).

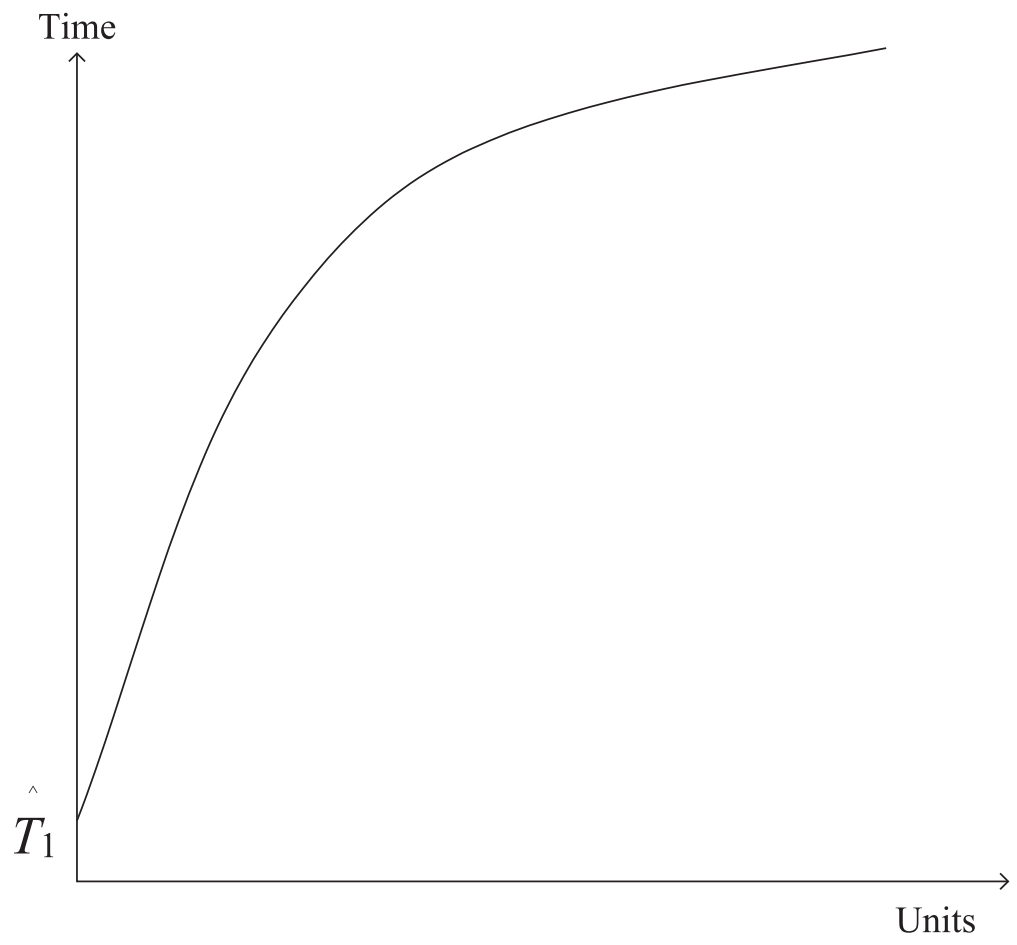

Figure 3.2: Forgetting curve

Jaber and Bonney (1998), Jaber and Bonney (1996), Jaber and Bonney (1997), and Jaber and Sikstrom (2004) report that the learn-forget curve model expresses the forget- 
ting exponent.

$$
f_{i}=\frac{l(1-l) \log \left(q_{i}+\alpha_{i}\right)}{\log \left(C_{i}+1\right)}
$$

where $q_{i}$ is the units produced in cycle $i, \alpha_{i}$ is the number of units of experience accumulated at the beginning of the cycle $i, t_{B}$ is the break time over which the total forgetting occurs, $t_{p i}$ is the time to accumulate $q_{i}$ units, and $C_{i}$ is the minimum ratio of the break time duration to the production time $\left(t_{B} / t_{p i}\right)$ as shown in Figure 3.3. Note that $f_{i}$ is zero whenever $l$ is 0 or 1 . It means that there will be no forgetting if there is no learning (nothing to forget) and rapid learning (forgetting slope unimportant (Jaber and Kher 2002)).

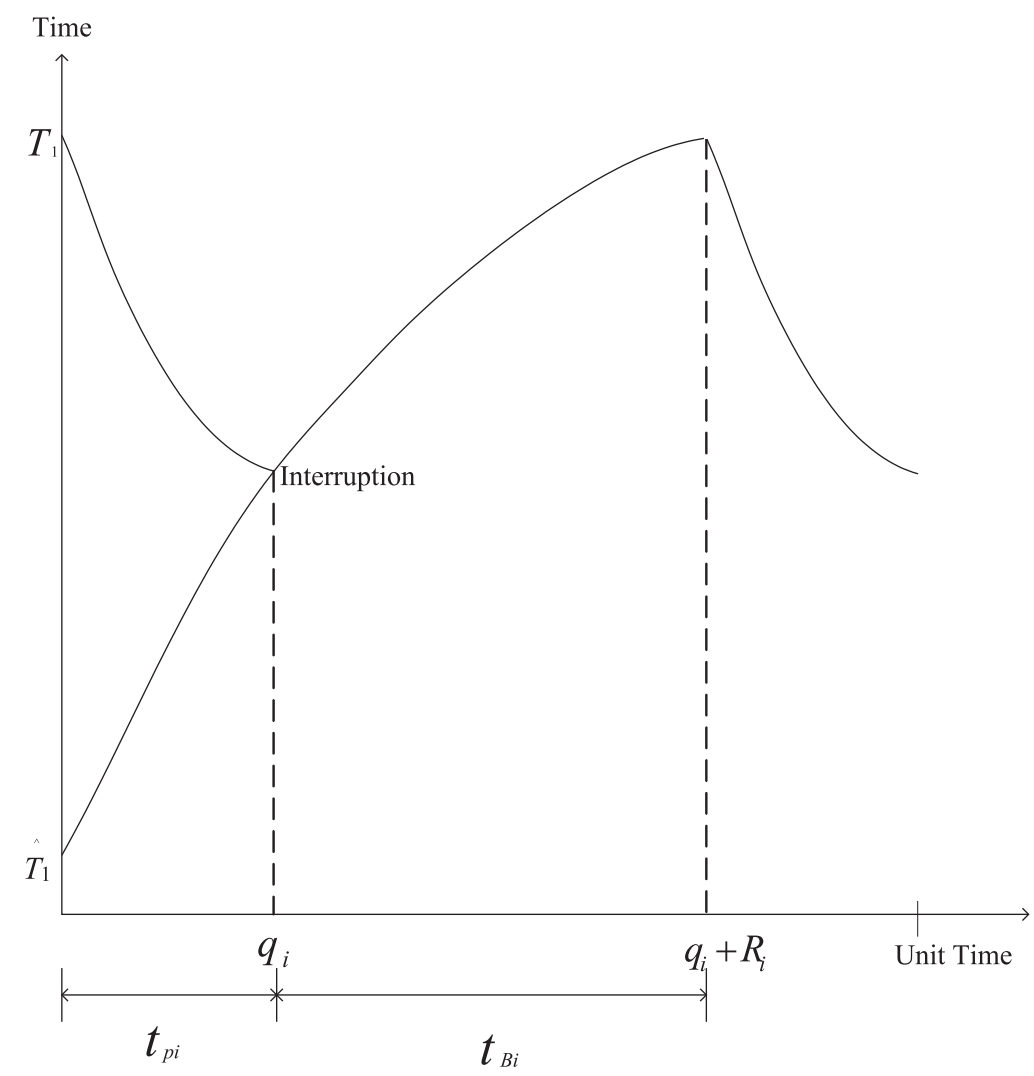

Figure 3.3: Learn-forget curve

If total forgetting occurs, then $\alpha_{i}$ is zero. If no forgetting occurs, then all units of accumulated experience from $i-1$ cycles are counted. Therefore, $\alpha_{i}$ is between 0 and $q_{n}$. 
The number of units accumulated for the next cycle, $\alpha_{i+1}$, is calculated as follows.

$$
\begin{gathered}
0<\alpha_{i}<\sum_{n=1}^{i-1} q_{n}, \\
\alpha_{i+1}=\left(q_{i}+\alpha_{i}\right)^{\left(l+f_{i}\right) / l}\left(q_{i}+R_{i}+\alpha_{i}\right)^{-f_{i} / l},
\end{gathered}
$$

where the $R_{i}$ is the number of units that would have been produced during the interruption period $t_{b i}$. The behavior of the learning-forget curve model is shown in Figure 3.3.

Once the time required to produce the first unit in first cycle is known, learning rate of the worker and the number of units accumulated experience at cycle $i+1$, the time required to produce the first unit for the cycle $i+1$ can be calculated as follows.

$$
T_{i+1}=T_{1}\left(\alpha_{i+1}+1\right)^{-l}
$$

There is a production plant and it manufactures in increasing production rate. The consumption rate is constant $r$ units per unit time as shown in Figure 3.4, where $Z_{i}$ is the maximum inventory level, $t_{c i}$ is the cycle time and it is equal to $t_{p i}$ and $t_{b i}, t_{p i}$ is the time required to produce $q_{i}$ units and build a maximum inventory of $Z_{i}, t_{b i}$ is the time to deplete $Z_{i}$. Note that $q_{i} \geq r t$ over the interval zero to $t_{p i}$. The inventory level as a function as per Figure 3.4 can be written as follows.

$$
\begin{gathered}
\phi_{i}(t)=q_{i}(t)-r t \quad\left(0 \leq t \leq t_{p i}\right) . \\
\phi_{i}(t)=-r t+r t_{c i} \quad\left(t_{p i} \leq t \leq t_{c i}\right) .
\end{gathered}
$$

The total time to produce $q_{i}$ units is $t\left(q_{i}\right) . T_{1+\alpha_{i}}$ is the time required to produce the first unit in cycle $i$. When the first unit is produced, there is already $\alpha_{i}$ units of accumulated experience from previous cycles. $T_{2+\alpha_{i}}$ is the time required to produce the second unit in cycle $i$ and so on. $T_{q_{i}+\alpha_{i}}$ is the time required to produce the $q_{i}^{\text {th }}$ unit in cycle $i$. By integrating $t\left(q_{i}\right)$ over the appropriate limits, the time required to produce $q_{i}$ is calculated as follows.

$$
t\left(q_{i}\right)=\int_{\alpha_{i}}^{q_{i}+\alpha_{i}} T_{1} x^{-l} d x,
$$




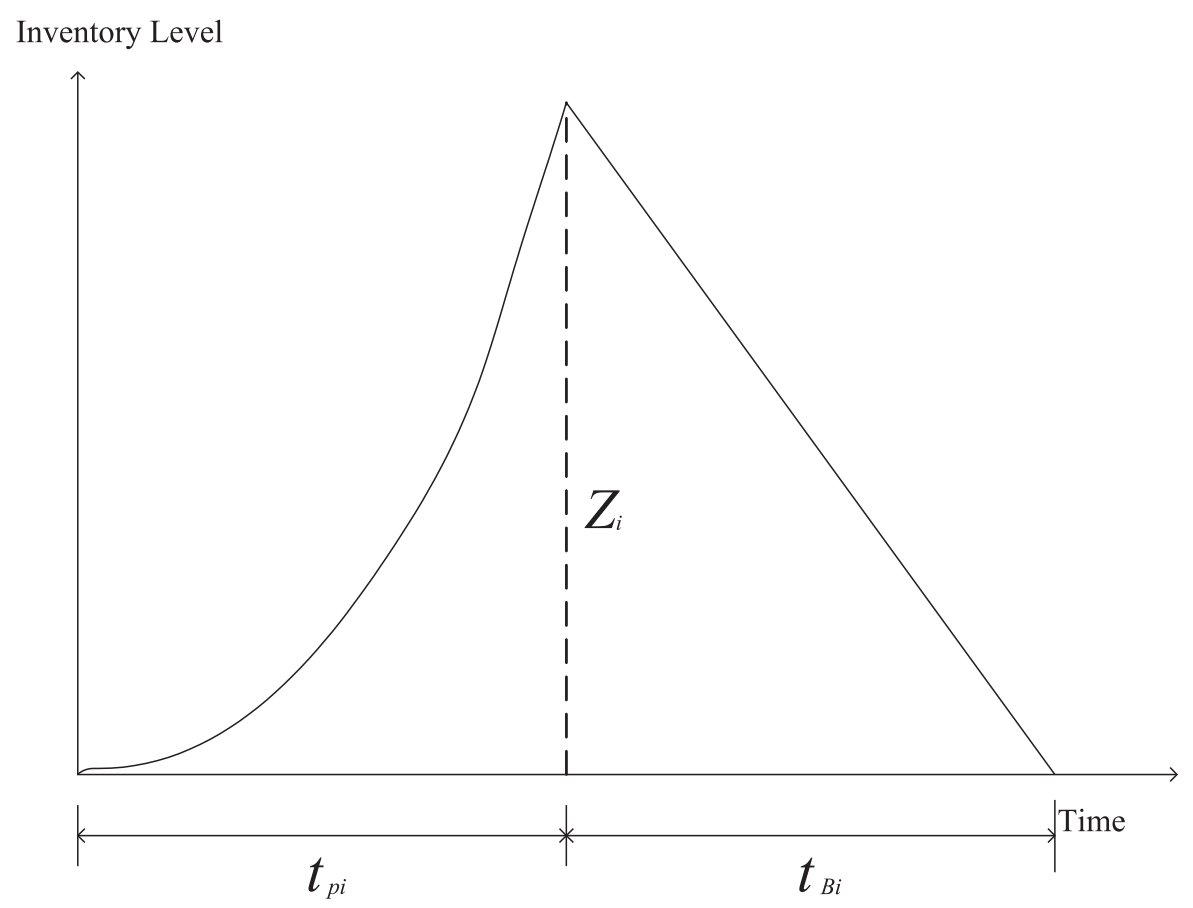

Figure 3.4: Inventory level affected by learning

\subsection{The deterministic learning curve model}

The deterministic learning curve model has its parameters, $T_{1}$ and $l$, given and are the same for every worker in a population of group of workers integrating $t\left(q_{i}\right)$ over the appropriate limits will give the time required to produce $q_{i}$ units. When $q_{i}$ is solved, it will give the quantity produced in function of time.

$$
\begin{aligned}
t\left(q_{i}\right) & =\frac{T_{1}}{(1-l)}\left[\left(q_{i}+\alpha_{i}\right)^{1-l}-\left(\alpha_{i}\right)^{1-l}\right] \\
q_{i} & =\left(\frac{1-l}{T_{1}} t+\alpha_{i}^{1-l}\right)^{(1 / 1-l)}-\alpha_{i} .
\end{aligned}
$$

\subsubsection{The infinite planning horizon model}

The infinite planning horizon model (Jaber and Bonney 1998) is presented to calculate total cost when the planning horizon is infinite, where $k$ is the setup cost, $h$ is the inventory carrying cost, $d_{m}$ is the material cost, and $\gamma$ is the labor cost. The expected 
total cost $(t c)$ equals to sum of production cost $(p c)$ and holding cost $(h c)$.

$$
\begin{aligned}
t c & =p c+h c . \\
p c & =k+d_{m} q_{i}+\gamma t\left(q_{i}\right) \\
& =k+d_{m} q_{i}+\frac{\gamma T_{1}}{1-l}\left[\left(q_{i}+\alpha_{i}\right)^{1-l}-\alpha_{i}^{1-l}\right] .
\end{aligned}
$$

Holding cost is inventory carrying cost $(h)$ per unit times the area under the inventory function in Figure 3.4. Thus, integrate $\phi_{i}(t)$ over the limit of 0 to $t_{c i}$ is given as follows.

$$
\int_{0}^{t_{c i}} \phi_{i}(t) d t=\frac{q_{i}^{2}}{2 r}-\frac{T_{1}\left[\left(q_{i}+\alpha_{i}\right)^{2-l}-\alpha_{i}^{2-l}\right]}{(1-l)(2-l)}+\frac{T_{1} q_{i} \alpha_{i}^{1-l}}{1-l} .
$$

After solving the area under the inventory function, the holding cost $(h c)$ is obtained by multiplying the area with the inventory carrying cost per unit $(h)$.

$$
h c=h\left(\frac{q_{i}^{2}}{2 r}-\frac{T_{1}\left[\left(q_{i}+\alpha_{i}\right)^{2-l}-\alpha_{i}^{2-l}\right]}{(1-l)(2-l)}\right)+\frac{h T_{1} q_{i} \alpha_{i}^{1-l}}{1-l} .
$$

Once the holding cost and the production cost are known, the total cost can be found by adding both costs. Therefore, the total cost is given as follows.

$$
\begin{aligned}
t c & =k+d_{m} q_{i}-\frac{\gamma T_{1}}{1-l}\left[\left(q_{i}+\alpha_{i}\right)^{1-l}+\alpha_{i}^{1-l}\right] \\
& +h\left(\frac{q_{i}^{2}}{2 r}-\frac{T_{1}\left[\left(q_{i}+\alpha_{i}\right)^{2-l}-\alpha_{i}^{2-l}\right]}{(1-l)(2-l)}\right)+\frac{h T_{1} q_{i} \alpha_{i}^{1-l}}{1-l} .
\end{aligned}
$$

The total cost per unit time can be found by dividing the total cost by cycle time. Therefore, the total cost per unit can be expressed as follows (Jaber and Bonney 1998).

$$
\begin{aligned}
\operatorname{tcu}\left(q_{i}\right) & =r t c\left(q_{i}\right) / q_{i} . \\
\operatorname{tcu}\left(q_{i}\right) & =\frac{k r}{q_{i}}+d_{m} r-\frac{\gamma T_{1} r}{1-l}\left(\frac{\left(q_{i}+\alpha_{i}\right)^{1-l}+\alpha_{i}^{1-l}}{q_{i}}\right) \\
& +h\left(\frac{q_{i}}{2}-\frac{T_{1} r\left[\left(q_{i}+\alpha_{i}\right)^{2-l}-\alpha_{i}^{2-l}\right]}{(1-l)(2-l) q_{i}}\right)+\frac{h T_{1} r \alpha_{i}^{1-l}}{1-l} .
\end{aligned}
$$

\subsubsection{The finite planning horizon model}

The finite planning horizon model (Jaber and Bonney 1998) is presented to calculate total cost when the planning horizon is finite, which means there is a finite demand to 
fulfill, where $k$ is the setup cost, $h$ is the inventory carrying cost, $d_{m}$ is the material cost, and $\gamma$ is the labor cost. The expected total cost $(t c)$ equals to sum of production cost $(p c)$ and holding cost $(h c)$.

When there are $N$ lots (a simplistic assumption of equal lots) and the total quantity demand is $Q$, where $Q=N q$, the total cost function can be calculated as in the infinite planning horizon model with some adjustments.

$$
\begin{aligned}
t c & =k N+d_{m} Q-\frac{\gamma T_{1}}{1-l}\left(\sum_{i=1}^{N}\left(\left(Q / N+\alpha_{i}\right)^{1-l}+\alpha_{i}^{1-l}\right)\right) \\
& +\frac{h Q^{2}}{2 N r}-\frac{T_{1}}{(1-l)(2-l)}\left(\sum_{i=1}^{N}\left(\left(Q / N+\alpha_{i}\right)^{2-l}-\alpha_{i}^{2-l}\right)\right)+\frac{h T_{1} Q}{N(1-l)}\left(\sum_{i=1}^{N}\left(\alpha_{i}^{1-l}\right)\right) .
\end{aligned}
$$

The optimal demand lots $N^{*}$ is in between $N_{L}$ and $N_{U}$. The total cost is calculated for all the lots in between $N_{L}$ and $N_{U}$ inclusive to find the actual $N^{*}$ with the minimum total cost.

\subsection{The probabilistic learning curve model}

In stochastic case, $T_{1}$ and $l$ are random variables as each worker in a population of workers has his/her learning curve and subsequently its own parameters. The learning curve parameters for the population of workers are treated as random variables with known pdfs. The new learning curve equation is as follows.

$$
t^{s}\left(q_{i}\right)=\int_{\alpha_{i}}^{q_{i}+\alpha_{i}} \int_{l_{a}}^{l_{b}} \int_{T_{1 a}}^{T_{1 b}} T_{1} x^{-l} f\left(T_{1}\right) f(l) d T_{1} d l d x
$$

where $t^{s}\left(q_{i}\right)$ is the total time to produce $q_{i}$ units in probabilistic learning curve equation. $T_{1 a}$ and $T_{1 b}$ are the limits for the time required to produce first unit, $T_{1} . l_{a}$ and $l_{b}$ are the limits for learning rate of each worker, $l$.

The functions $f\left(T_{1}\right)$ and $f(l)$ are any function in general for stochastic $T_{1}$ and $l$. For simplicity, this study assumes that $T_{1}$ and $l$ are using uniformly distributed given 
respectively as

$$
f\left(T_{1}\right)=\frac{1}{\left(T_{1 b}-T_{1 a}\right)},
$$

and

$$
f(l)=\frac{1}{\left(l_{b}-l_{a}\right)} .
$$

By integrating the learning curve in Equation (3.17) over the proper limits, the expected time required to produce $q_{i}$ units, $t^{s}\left(q_{i}\right)$, is given as,

$$
\begin{aligned}
t^{s}\left(q_{i}\right) & =\int_{\alpha_{i}}^{q_{i}+\alpha_{i}} \int_{l_{a}}^{l_{b}} \int_{T_{1 a}}^{T_{1 b}} T_{1} x^{-l} \frac{1}{\left(T_{1 b}-T_{1 a}\right)} \frac{1}{\left(l_{b}-l_{a}\right)} d T_{1} d l d x \\
& =-\frac{\left(T_{1 b}+T_{1 a}\right)}{2\left(l_{b}-l_{a}\right)} \int_{\alpha_{i}}^{q_{i}+\alpha_{i}}\left(\frac{x^{-l_{b}}}{\ln (x)}-\frac{x^{-l_{a}}}{\ln (x)}\right) d x,
\end{aligned}
$$

where $T_{1 a}$ and $T_{1 b}$ are the limits for the time required to produce first unit, $T_{1} \cdot l_{a}$ and $l_{b}$ are the limits for learning rate of each worker, $l$. As for the deterministic case, the total time to produce $q_{i}$ units is defined with time required to produce the first unit in cycle $i$ plus the time required to produce the second units and so on until the time required to produce the $q_{i}^{t h}$ unit is added and integrated over the limit $\alpha_{i}$ and $q_{i}+\alpha_{i}$, the time to produce $q_{i}$ units can be calculated. Derivation of Equation (3.20) is given in Appendix A and it is solved using Matlab for the numerical results.

Equation (3.17) can be approximated as follows:

$$
\begin{aligned}
t_{e}^{s}\left(q_{i}\right)= & \int_{\alpha_{i}}^{q_{i}+\alpha_{i}} \int_{l_{a}}^{l_{b}} \int_{T_{1 a}}^{T_{1 b}} T_{1} x^{-l} \frac{1}{\left(T_{1 b}-T_{1 a}\right)} \frac{1}{\left(l_{b}-l_{a}\right)} d T_{1} d l d x \\
= & -\frac{\left(T_{1 b}+T_{1 a}\right)}{2\left(l_{b}-l_{a}\right)} \int_{\alpha_{i}}^{q_{i}+\alpha_{i}}\left(\frac{x^{-l_{b}}}{\ln (x)}-\frac{x^{-l_{a}}}{\ln (x)}\right) d x \\
\approx & -\frac{\left(T_{1 b}+T_{1 a}\right)}{2\left(l_{b}-l_{a}\right)} \int_{\alpha_{i}}^{q_{i}+\alpha_{i}}\left(\lambda_{b} x^{-\beta_{b}}-\lambda_{a} x^{-\beta_{a}}\right) d x \\
\approx & -\frac{\left(T_{1 b}+T_{1 a}\right)}{2\left(l_{b}-l_{a}\right)} \\
& \quad\left\{\frac{\lambda_{b}}{1-\beta_{b}}\left[\left(q_{i}+\alpha_{i}\right)^{1-\beta_{b}}-\left(\alpha_{i}\right)^{1-\beta_{b}}\right]-\frac{\lambda_{a}}{1-\beta_{a}}\left[\left(q_{i}+\alpha_{i}\right)^{1-\beta_{a}}-\left(\alpha_{i}\right)^{1-\beta_{a}}\right]\right\},
\end{aligned}
$$

where $\lambda_{a}=0.604743-0.065762 l_{a}, \beta_{a}=0.21264+0.981904762 l_{a}, \lambda_{b}=0.604743-$ $0.065762 l_{b}$ and $\beta_{b}=0.21264+0.981904762 l_{b}$ 


\subsubsection{The infinite planning horizon model}

The infinite planning horizon model is presented to calculate total cost with stochastic functions when the planning horizon is infinite. That is there is no finite demand requested. Using the new stochastic time function, the total cost function can be calculated. Define $k$ as the setup cost, $h$ as the inventory carrying cost, $d_{m}$ as the material cost, and $\gamma$ as the labor cost. The expected total cost $(t c)$ equals to sum of production cost $(p c)$ and holding cost $(h c)$.

$$
\begin{aligned}
t c & =p c+h c . \\
p c & =k+d_{m} q_{i}+\gamma t^{s}\left(q_{i}\right) \\
& =k+d_{m} q_{i}-\gamma\left[\frac{\left(T_{1 b}+T_{1 a}\right)}{2\left(l_{b}-l_{a}\right)} \int_{\alpha_{i}}^{q_{i}+\alpha_{i}}\left(\frac{x^{-l_{b}}}{\ln (x)}-\frac{x^{-l_{a}}}{\ln (x)}\right) d x\right] .
\end{aligned}
$$

Equation (3.22) is solved using Matlab for the numerical results. If the approximated equation is substituted for the above integration, the approximated production cost is given as follows.

$$
\begin{aligned}
p c_{e}= & k+d_{m} q_{i}+\gamma t^{s}\left(q_{i}\right) \\
= & k+d_{m} q_{i}-\left[\frac{\gamma\left(T_{1 b}+T_{1 a}\right)}{2\left(l_{b}-l_{a}\right)}\right] \\
& \left\{\frac{\lambda_{b}}{1-\beta_{b}}\left[\left(q_{i}+\alpha_{i}\right)^{1-\beta_{b}}-\left(\alpha_{i}\right)^{1-\beta_{b}}\right]-\frac{\lambda_{a}}{1-\beta_{a}}\left[\left(q_{i}+\alpha_{i}\right)^{1-\beta_{a}}-\left(\alpha_{i}\right)^{1-\beta_{a}}\right]\right\} .
\end{aligned}
$$

Holding cost is the inventory carrying cost, $h$, times the area under the inventory function. It is very hard to integrate stochastic time function to get the area under the inventory function, so it is approximated as Salameh et al. (1993) defined. The average inventory level over $t_{c i}$ is given as follows.

$$
\phi_{i}(t)=\frac{q_{i}}{2 r}\left(r t^{s}\left(q_{i}\right)\right)-q_{i}-\frac{\left(l_{b}+l_{a}\right) q_{i} t^{s}\left(q_{i}\right)}{\left(2\left(4-l_{b}+l_{a}\right)\right)}
$$

where $\frac{1}{T_{1 a}}>r$ 
After solving the area under the inventory function, the holding cost $(h c)$ is found by multiplying the area with the inventory carrying cost, $h$.

$$
\begin{aligned}
h c & =h \phi_{i}(t) . \\
h c & =\frac{h q_{i} t^{s}\left(q_{i}\right)}{2}-\frac{h q_{i}^{2}}{2 r}-\frac{h\left(l_{b}+l_{a}\right) q_{i} t^{s}\left(q_{i}\right)}{\left(2\left(4-l_{b}+l_{a}\right)\right)} .
\end{aligned}
$$

Once the holding cost and the production cost are known, the total cost can be found by adding both costs. Therefore, the total cost is given as follows.

$$
\begin{aligned}
t c= & k+d_{m} q_{i}+\gamma t^{s}\left(q_{i}\right)-\frac{h q_{i}^{2}}{2 r}+\frac{h q_{i} t^{s}\left(q_{i}\right)}{2}-\frac{h\left(l_{b}+l_{a}\right) q_{i} t^{s}\left(q_{i}\right)}{\left(2\left(4-l_{b}+l_{a}\right)\right)} \\
= & k+d_{m} q_{i}-\frac{h q_{i}^{2}}{2 r}+\left[\gamma+\frac{h q_{i}}{2}-\frac{h\left(l_{b}+l_{a}\right) q_{i}}{\left(2\left(4-l_{b}+l_{a}\right)\right)}\right] \\
& {\left[-\frac{\left(T_{1 b}+T_{1 a}\right)}{2\left(l_{b}-l_{a}\right)} \int_{\alpha_{i}}^{q_{i}+\alpha_{i}}\left(\frac{x^{-l_{b}}}{\ln (x)}-\frac{x^{-l_{a}}}{\ln (x)}\right) d x\right] . }
\end{aligned}
$$

This equation can be approximated as follows:

$$
\begin{aligned}
t c_{e}= & k+d_{m} q_{i}+\gamma t^{s}\left(q_{i}\right)-\frac{h q_{i}^{2}}{2 r}+\frac{h q_{i} t^{s}\left(q_{i}\right)}{2}-\frac{h\left(l_{b}+l_{a}\right) q_{i} t^{s}\left(q_{i}\right)}{\left(2\left(4-l_{b}+l_{a}\right)\right)} \\
= & k+d_{m} q_{i}-\frac{h q_{i}^{2}}{2 r}+\left[\gamma+\frac{h q_{i}}{2}-\frac{h\left(l_{b}+l_{a}\right) q_{i}}{\left(2\left(4-l_{b}+l_{a}\right)\right)}\right]\left[-\frac{\left(T_{1 b}+T_{1 a}\right)}{2\left(l_{b}-l_{a}\right)}\right] \\
& \left\{\frac{\lambda_{b}}{1-\beta_{b}}\left[\left(q_{i}+\alpha_{i}\right)^{1-\beta_{b}}-\left(\alpha_{i}\right)^{1-\beta_{b}}\right]-\frac{\lambda_{a}}{1-\beta_{a}}\left[\left(q_{i}+\alpha_{i}\right)^{1-\beta_{a}}-\left(\alpha_{i}\right)^{1-\beta_{a}}\right]\right\} .
\end{aligned}
$$

From the total cost function, the expected total cost per unit $\left(t c u\left(q_{i}\right)\right)$ can be calculated by dividing the total cost by cycle time as follows,

$$
\begin{aligned}
\operatorname{tcu}\left(q_{i}\right)= & \frac{t c\left(q_{i}\right)}{t_{c i}} . \\
\operatorname{tcu}\left(q_{i}\right)= & t c\left(q_{i}\right) \frac{r}{q_{i}} . \\
t c u\left(q_{i}\right)= & \frac{k r}{q_{i}}+r d_{m}-\frac{h q_{i}}{2}+\left[\frac{\gamma r}{q_{i}}+\frac{h r}{2}-\frac{h\left(l_{b}+l_{a}\right) r}{\left(2\left(4-l_{b}+l_{a}\right)\right)}\right] \\
& {\left[-\frac{\left(T_{1 b}+T_{1 a}\right)}{2\left(l_{b}-l_{a}\right)} \int_{\alpha_{i}}^{q_{i}+\alpha_{i}}\left(\frac{x^{-l_{b}}}{\ln (x)}-\frac{x^{-l_{a}}}{\ln (x)}\right) d x\right] . }
\end{aligned}
$$


If the approximated equation is substituted for the above integration, the approximated expected total cost per unit is given as follows.

$$
\begin{aligned}
t c u_{e}\left(q_{i}\right)= & \frac{t c\left(q_{i}\right)}{t_{c i}} . \\
t c u_{e}\left(q_{i}\right)= & t c\left(q_{i}\right) \frac{r}{q_{i}} . \\
t c u_{e}\left(q_{i}\right)= & \frac{k r}{q_{i}}+r d_{m}-\frac{h q_{i}}{2}+\left[\frac{\gamma r}{q_{i}}+\frac{h r}{2}-\frac{h\left(l_{b}+l_{a}\right) r}{\left(2\left(4-l_{b}+l_{a}\right)\right)}\right]\left[-\frac{\left(T_{1 b}+T_{1 a}\right)}{2\left(l_{b}-l_{a}\right)}\right] \\
& \left\{\frac{\lambda_{b}}{1-\beta_{b}}\left[\left(q_{i}+\alpha_{i}\right)^{1-\beta_{b}}-\left(\alpha_{i}\right)^{1-\beta_{b}}\right]-\frac{\lambda_{a}}{1-\beta_{a}}\left[\left(q_{i}+\alpha_{i}\right)^{1-\beta_{a}}-\left(\alpha_{i}\right)^{1-\beta_{a}}\right]\right\} .
\end{aligned}
$$

\subsubsection{The finite planning horizon model}

The finite planning horizon model is to calculate total cost with stochastic time function when the planning horizon is finite. That is, a finite demand is requested in the production plant. The total cost function is expressed with $k$ as the setup cost, $h$ as the inventory carrying cost, $d_{m}$ as the material cost, and $\gamma$ as the labor cost. The expected total cost $(t c)$ equals to sum of production cost $(p c)$ and holding cost $(h c)$.

When there are $N$ lots and the total quantity demand is $Q$, where $Q=N q$, the total cost is calculated with some adjustments to the infinite planning horizon model.

$$
\begin{aligned}
t c= & k N+d_{m} Q-\frac{h Q^{2}}{2 N r}+\left[\gamma+\frac{h Q}{2 N}-\frac{h\left(l_{b}+l_{a}\right) Q}{\left(2 N\left(4-l_{b}+l_{a}\right)\right)}\right]\left[-\frac{\left(T_{1 b}+T_{1 a}\right)}{2\left(l_{b}-l_{a}\right)}\right] \\
& \sum_{i=1}^{N}\left[\int_{\alpha_{i}}^{Q / N+\alpha_{i}}\left(\frac{x^{-l_{b}}}{\ln (x)}-\frac{x^{-l_{a}}}{\ln (x)}\right) d x\right] .
\end{aligned}
$$

If the approximated equation is substituted for the above integration, the approximated total cost to the infinite planning horizon model is given as follows.

$$
\begin{aligned}
t c_{e}= & k N+d_{m} Q-\frac{h Q^{2}}{2 N r}+\left[\gamma+\frac{h Q}{2 N}-\frac{h\left(l_{b}+l_{a}\right) Q}{\left(2 N\left(4-l_{b}+l_{a}\right)\right)}\right]\left[-\frac{\left(T_{1 b}+T_{1 a}\right)}{2\left(l_{b}-l_{a}\right)}\right] \\
& \sum_{i=1}^{N}\left[\frac{\lambda_{b}}{1-\beta_{b}}\left[\left(Q / N+\alpha_{i}\right)^{1-\beta_{b}}-\left(\alpha_{i}\right)^{1-\beta_{b}}\right]-\frac{\lambda_{a}}{1-\beta_{a}}\left[\left(Q / N+\alpha_{i}\right)^{1-\beta_{a}}-\left(\alpha_{i}\right)^{1-\beta_{a}}\right]\right] .
\end{aligned}
$$




\subsection{Numerical example and analysis}

Let us assume there is a production line and its inventory is affected with learning and forgetting. Consider the $T_{1}$ (time to produce the first unit) vary from 0.050 to 0.075 days per unit (Jaber and Bonney 1998). The consumption rate $(r)$ is 12 units per day. The labor cost $(\gamma)$ is $\$ 80$ per day. The holding cost $(h)$ is $\$ 0.20$ per day. The set up cost $(k)$ is $\$ 200$ per production run. The learning curve slope varies from 0.05 to 0.15 to get $l=$

0.1 as Jaber and Bonney (1998) assumed. Assume $t_{B}$ is 300 days; that is the length of the interruption or break period where the total forgetting occurs. The same experiment is repeated when $t_{B}$ is equal to $500,1000,3000,6000$, and 12000 days.

For the stochastic case, using the above numerical values, we calculate optimum production quantities for the cycles $1,23, \ldots, 12$ (see Table 3.1). The optimum quantity with $t_{B}=300$ is 465 in cycle 1 .

We need to find the output, that would have been build up if an interruption did not occur at unit number 465 in first cycle, to find the level experience at second cycle, $\alpha_{2}$. To find this, we need $t_{c 1}$, which is the time of the first cycle. The amount of time, $t_{c 1}$, required to produce 465 units can be calculated from Equation $(3.24)$, is $t(465)=17.65$ day. Matlab is used to calculate the numerical value.

We need to determine the level of experience at the beginning of the second cycle. For this we have to find the output that would have been collected if no interruption occurred at unit 465. We use the following equation to find the total output (Jaber and Bonney 1998):

$$
\begin{aligned}
\alpha_{1}+q_{1}+R_{1} & =\left[\frac{1-0.1}{0.0625}\left(\frac{465}{12}\right)+0^{1-0.1}\right]^{1 / 1-0.1} \\
& =1126 \text { units }
\end{aligned}
$$


The forgetting slope is calculated as follows (Jaber and Bonney 1998):

$$
\begin{aligned}
f_{i} & =\frac{0.1(1-0.1) \log (465+0)}{\log (16.99+1)} \\
& =0.1913
\end{aligned}
$$

where $C_{i}$ is $t_{B} / t(465)$ and $t_{B}$ is 300 days.

For the deterministic case with $T_{1}$ is 0.0625 and $l$ is 0.1 , the optimum quantity with $t_{B}=300$ is 248 in cycle 1 . The amount of time, $t_{c 1}$, required to produce 248 units is 9.92 days. The total output, when no interruption occurred at unit 248, is 560 units and the forgetting slope is 0.1442. The total cost is 60.40 (Jaber and Bonney 1998).

Two extreme cases for deterministic learning curve are found as follows: (1) very fast ( $T_{1 b}$ is 0.05 and $l_{b}$ is 0.15$)$ and (2) very slow $\left(T_{1 a}\right.$ is 0.075 and $l_{a}$ is 0.05$)$. For the first case, the optimum quantity with $t_{B}=300$ is 213 in cycle 1 . The amount of time, $t_{c 1}$, required to produce 213 units is 5.59 days. The total output, when no interruption occurred at unit 213 , is 825 units and the forgetting slope is 0.1709 . The total cost is 50.61 . For the second case, the optimum quantity with $t_{B}=300$ is 328 in cycle 1 . The amount of time, $t_{c 1}$, required to produce 328 units is 19.39 days. The total output, when no interruption occurred at unit 328, is 472 units and the forgetting slope is 0.0983 . The total cost is 73.02 .

Then, the process is repeated for $t_{B}$ is equal to 500 days. The second part of Table 3.1 shows the results for $t_{B}$ is equal to 500 days. The optimum quantities produced are 465, 436, 434, 433, 430, 429, 429, 429, 429, 429, 429, and 429 for cycles 1-12 respectively. The estimated optimum quantities produced are 481, 451, 449, 448, 445, 444, 444, 444, 444, 444, 444, and 444 for cycles 1-12 respectively.

Then, the process is repeated for $t_{B}$ is equal to 1000 days. The first part of Table 3.2 shows the results for $t_{B}$ is equal to 1000 days. The optimum quantities produced are $465,435,433,432,431,429,427,427,427,427,427$, and 427 for cycles 1-12 respectively. The estimated optimum quantities produced are 481, 450, 448, 447, 446, 444, 442, 442, 
$442,442,442$, and 442 for cycles 1-12 respectively.

Then, the process is repeated for $t_{B}$ is equal to 3000 days. The second part of Table 3.2 shows the results for $t_{B}$ is equal to 3000 days. The optimum quantities produced are $465,433,432,430,427,426,425,425,425,425,425$, and 425 for cycles 1-12 respectively. The estimated optimum quantities produced are 481, 448, 447, 445, 442, 440, 439, 439, 439, 439, 439, and 439 for cycles 1-12 respectively.

Then, the process is repeated again for $t_{B}$ is equal to 6000 days. The first part of Table 3.3 shows the results for $t_{B}$ is equal to 6000 days. The optimum quantities produced are $465,430,427,424,420,420,420,420,420,420,420$, and 420 for cycles 1-12 respectively. The estimated optimum quantities produced are 481, 445, 442, 438, 434, 434, 434, 434, $434,434,434$, and 434 for cycles $1-12$ respectively.

Then, the process is repeated again for $t_{B}$ is equal to 12000 days. The second part of Table 3.3 shows the results for $t_{B}$ is equal to 12000 days. The optimum quantities produced are 465, 430, 425, 423, 420, 420, 420, 420, 420, 420, 420, and 420 for cycles 1-12 respectively. The estimated optimum quantities produced are 481, 445, 439, 437, 434, 434, 434, 434, 434, 434, 434, and 434 for cycles 1-12 respectively.

Comparing the results obtained for $t_{B}=300,500,1000,3000,6000$, and 12000 days, the cost per unit time was reduced from $66.69,65.82,64.82,63.47,62.41$ to 61.77 time units, where the production is rate increased as a consequence. The optimum quantities that were produced after the first cycle are decreased. This indicates that the longer it takes to forget after a break, the less would be the impact of forgetting on the total cost and optimum quantity produced. When we increase $t_{B}$ toward infinity, the changes in optimum quantity produced is very minimal. This happens because when the break between two cycles increases, the workers intend to forget more and more. Once the limit is reached, they fully forget and need to learn from the beginning. In this case, you can see they fully forgot when $t_{B}=6000$, therefore, there is no change in quantity produced when $t_{B}$ is changed to 12000 . 
The approximated Equation (3.21) is calculated when the upper and lower limits of the learning curve slope, $l_{b}=0.4$ and $l_{a}=0.1$, respectively. The approximated result for the time request to produce 100 units is 1.82 days when the number of units accumulated at the beginning of the cycle is 200.5. The result from Equation (3.20) is 1.76 days for the same parameters. Therefore, the error is $(1.82-1.76) / 1.76=0.034$.

Table 3.1: Optimum quantity and estimated optimum quantity produced for 12 cycles for $t_{B}=300$ and 500 days, respectively

\begin{tabular}{|l|c|c|c|c|c|c|c|}
\hline Cycle $_{i}$ & $\alpha_{i}$ & $q$ & $t\left(q_{i}\right)$ & Total cost & $q_{e}$ & $t_{e}\left(q_{i}\right)$ & Estimated cost \\
\hline \hline 1 & 0 & 465 & 17.65 & 73.25 & 481 & 18.25 & 75.74 \\
2 & 86.82 & 438 & 15.83 & 67.66 & 453 & 16.37 & 69.96 \\
3 & 97.18 & 433 & 15.76 & 67.46 & 448 & 16.29 & 69.75 \\
4 & 98.78 & 432 & 15.59 & 66.93 & 447 & 16.12 & 69.20 \\
5 & 99.22 & 431 & 15.58 & 66.91 & 446 & 16.10 & 69.18 \\
6 & 99.27 & 431 & 15.55 & 66.80 & 446 & 16.07 & 69.07 \\
7 & 99.31 & 431 & 15.51 & 66.70 & 446 & 16.03 & 68.96 \\
8 & 99.33 & 431 & 15.51 & 66.69 & 446 & 16.03 & 68.95 \\
9 & 99.33 & 431 & 15.51 & 66.69 & 446 & 16.03 & 68.95 \\
10 & 99.33 & 431 & 15.51 & 66.69 & 446 & 16.03 & 68.95 \\
11 & 99.33 & 431 & 15.51 & 66.69 & 446 & 16.03 & 68.95 \\
12 & 99.33 & 431 & 15.51 & 66.69 & 446 & 16.03 & 68.95 \\
\hline 1 & 0 & 465 & 17.65 & 73.25 & 481 & 18.25 & 75.74 \\
2 & 110.59 & 436 & 15.62 & 66.99 & 451 & 16.15 & 69.26 \\
3 & 129.94 & 434 & 15.44 & 66.44 & 449 & 15.96 & 68.69 \\
4 & 134.24 & 433 & 15.38 & 66.26 & 448 & 15.90 & 68.51 \\
5 & 135.18 & 430 & 15.28 & 65.93 & 445 & 15.79 & 68.17 \\
6 & 135.41 & 429 & 15.24 & 65.82 & 444 & 15.75 & 68.05 \\
7 & 135.45 & 429 & 15.24 & 65.82 & 444 & 15.75 & 68.04 \\
8 & 135.45 & 429 & 15.24 & 65.82 & 444 & 15.75 & 68.04 \\
9 & 135.46 & 429 & 15.24 & 65.82 & 444 & 15.75 & 68.04 \\
10 & 135.46 & 429 & 15.24 & 65.82 & 444 & 15.75 & 68.04 \\
11 & 135.46 & 429 & 15.24 & 65.82 & 444 & 15.75 & 68.04 \\
12 & 135.46 & 429 & 15.24 & 65.82 & 444 & 15.75 & 68.04 \\
\hline
\end{tabular}

Not all the workers have the same learning rate or do the work in same time frame. 
Table 3.2: Optimum quantity and estimated optimum quantity produced for 12 cycles for $t_{B}=1000$ and 3000 days, respectively

\begin{tabular}{|l|c|c|c|c|c|c|c|}
\hline Cycle $_{i}$ & $\alpha_{i}$ & $q$ & $t\left(q_{i}\right)$ & Total cost & $q_{e}$ & $t_{e}\left(q_{i}\right)$ & Estimated cost \\
\hline \hline 1 & 0 & 465 & 17.65 & 73.25 & 481 & 18.25 & 75.74 \\
2 & 140.44 & 435 & 15.43 & 66.40 & 450 & 15.95 & 68.66 \\
3 & 175.40 & 433 & 15.20 & 65.67 & 448 & 15.72 & 67.90 \\
4 & 186.23 & 432 & 15.12 & 65.42 & 447 & 15.63 & 67.64 \\
5 & 189.60 & 431 & 15.07 & 65.26 & 446 & 15.58 & 67.48 \\
6 & 190.63 & 429 & 15.00 & 65.04 & 444 & 15.51 & 67.25 \\
7 & 190.88 & 427 & 14.93 & 64.83 & 442 & 15.44 & 67.04 \\
8 & 190.87 & 427 & 14.93 & 64.82 & 442 & 15.44 & 67.02 \\
9 & 190.86 & 427 & 14.93 & 64.82 & 442 & 15.44 & 67.02 \\
10 & 190.84 & 427 & 14.93 & 64.82 & 442 & 15.44 & 67.02 \\
11 & 190.84 & 427 & 14.93 & 64.82 & 442 & 15.44 & 67.02 \\
12 & 190.83 & 427 & 14.93 & 64.82 & 442 & 15.44 & 67.02 \\
\hline 1 & 0 & 465 & 17.65 & 73.25 & 481 & 18.25 & 75.74 \\
2 & 180.82 & 433 & 15.18 & 65.60 & 448 & 15.70 & 67.83 \\
3 & 244.20 & 432 & 14.91 & 64.72 & 447 & 15.42 & 66.92 \\
4 & 271.46 & 430 & 14.75 & 64.22 & 445 & 15.25 & 66.40 \\
5 & 283.20 & 427 & 14.61 & 63.77 & 442 & 15.11 & 65.94 \\
6 & 288.05 & 426 & 14.56 & 63.61 & 440 & 15.06 & 65.77 \\
7 & 290.08 & 425 & 14.52 & 63.48 & 439 & 15.01 & 65.64 \\
8 & 290.87 & 425 & 14.51 & 63.48 & 439 & 15.00 & 65.64 \\
9 & 291.22 & 425 & 14.51 & 63.47 & 439 & 15.00 & 65.63 \\
10 & 291.38 & 425 & 14.51 & 63.47 & 439 & 15.00 & 65.63 \\
11 & 291.45 & 425 & 14.51 & 63.47 & 439 & 15.00 & 65.63 \\
12 & 291.48 & 425 & 14.51 & 63.47 & 439 & 15.00 & 65.63 \\
\hline
\end{tabular}

Even one worker does not always have the ability to finish one product in the same time frame over and over again. Thus, we gave stochastic learning rate and time to do the units. It makes the process more realistic. It is shown that there is a significant difference when we give stochastic characteristics for workers to model the learning curve instead of deterministic characteristics. The numerical example using uniform distribution for both learning curve slope and the time to produce first unit shows differences. 
Table 3.3: Optimum quantity and estimated optimum quantity produced for 12 cycles for $t_{B}=6000$ and 12000 days, respectively

\begin{tabular}{|c|c|c|c|c|c|c|c|}
\hline Cycle $_{i}$ & $\alpha_{i}$ & $q$ & $t\left(q_{i}\right)$ & Total cost & $q_{e}$ & $t_{e}\left(q_{i}\right)$ & Estimated cost \\
\hline 1 & 0 & 465 & 17.65 & 73.25 & 481 & 18.25 & 75.74 \\
\hline 2 & 202.17 & 430 & 15.00 & 65.01 & 445 & 15.51 & 67.22 \\
\hline 3 & 283.33 & 427 & 14.62 & 63.80 & 442 & 15.12 & 66.00 \\
\hline 4 & 322.92 & 424 & 14.41 & 63.14 & 438 & 14.90 & 65.29 \\
\hline 5 & 342.39 & 420 & 14.23 & 62.56 & 434 & 14.71 & 64.68 \\
\hline 6 & 238.39 & 420 & 14.21 & 62.48 & 434 & 14.69 & 64.60 \\
\hline 7 & 351.69 & 420 & 14.20 & 62.45 & 434 & 14.68 & 64.57 \\
\hline 8 & 356.43 & 420 & 14.19 & 62.43 & 434 & 14.67 & 64.57 \\
\hline 9 & 358.85 & 420 & 14.19 & 62.42 & 434 & 14.67 & 64.57 \\
\hline 10 & 360.10 & 420 & 14.19 & 62.42 & 434 & 14.67 & 64.57 \\
\hline 11 & 360.73 & 420 & 14.19 & 62.41 & 434 & 14.67 & 64.57 \\
\hline 12 & 361.06 & 420 & 14.19 & 62.41 & 434 & 14.67 & 64.57 \\
\hline 1 & 0 & 465 & 17.65 & 73.25 & 481 & 18.25 & 75.74 \\
\hline 2 & 220.81 & 430 & 14.93 & 64.78 & 445 & 15.44 & 66.98 \\
\hline 3 & 320.09 & 425 & 14.45 & 63.24 & 439 & 14.94 & 65.39 \\
\hline 4 & 374.05 & 423 & 14.25 & 62.58 & 437 & 14.93 & 64.70 \\
\hline 5 & 403.749 & 420 & 14.08 & 61.92 & 434 & 14.56 & 64.02 \\
\hline 6 & 419.79 & 420 & 14.04 & 61.85 & 434 & 14.52 & 63.95 \\
\hline 7 & 428.99 & 420 & 14.02 & 61.81 & 434 & 14.50 & 63.91 \\
\hline 8 & 434.29 & 420 & 14.01 & 61.79 & 434 & 14.48 & 63.89 \\
\hline 9 & 437.36 & 420 & 14.00 & 61.78 & 434 & 14.47 & 63.88 \\
\hline 10 & 439.14 & 420 & 14.00 & 61.77 & 434 & 14.47 & 63.87 \\
\hline 11 & 440.18 & 420 & 14.00 & 61.77 & 434 & 14.47 & 63.87 \\
\hline 12 & 440.78 & 420 & 14.00 & 61.77 & 434 & 14.47 & 63.87 \\
\hline
\end{tabular}

We can see the changes in time to produce quantity when we change the upper limit of the initial processing time $T_{1 b}$ as shown in Figure 3.5. The bold line is for the deterministic case and the others are for probabilistic cases. In here, when the upper limit of the initial processing time is increased, the time to produce the quantity is increased.

We can see the changes in time to produce quantity when we change the lower limit of 


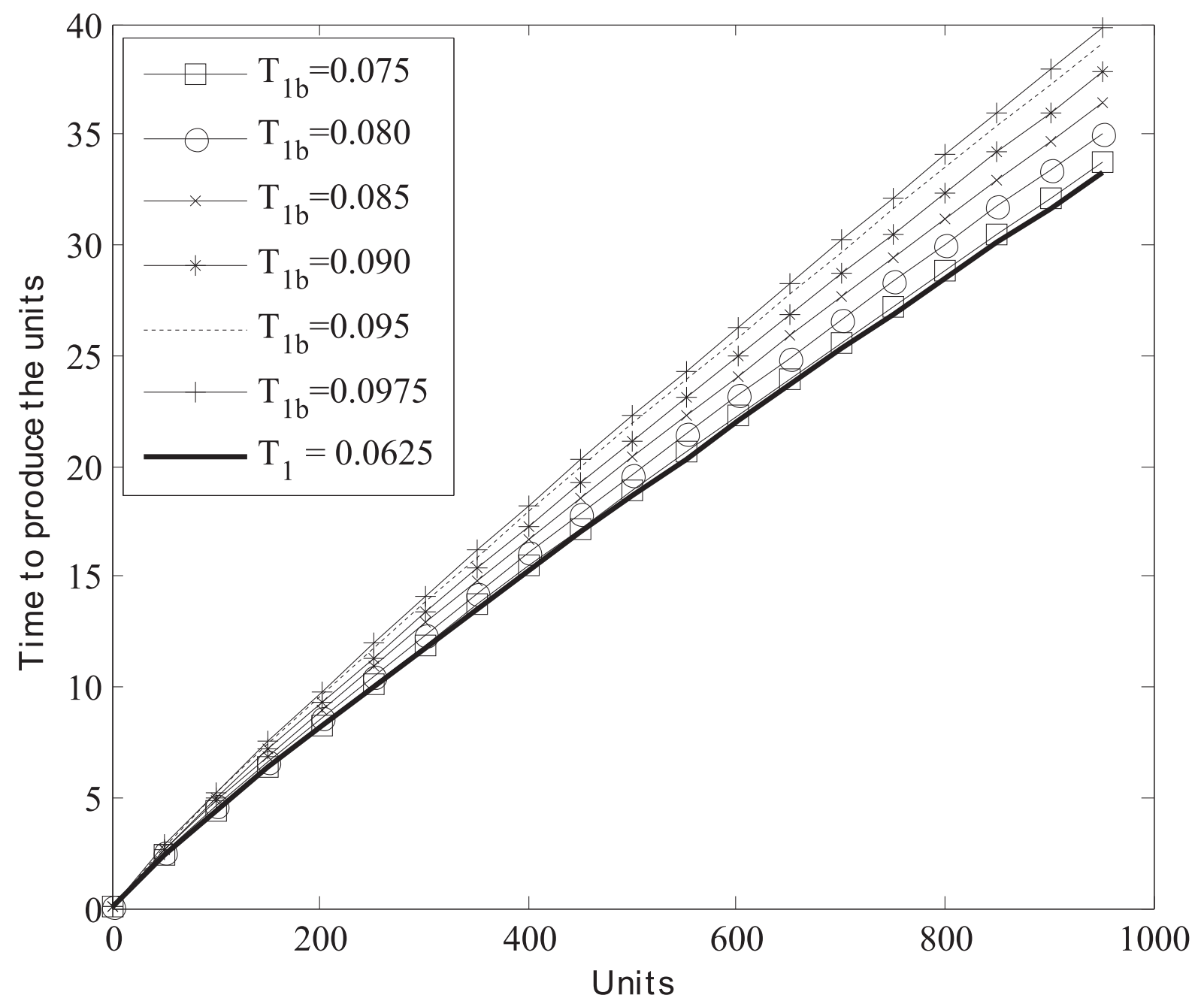

Figure 3.5: Effect on the output when $T_{1 b}$ changes

the initial processing time $T_{1 a}$ as shown in Figure 3.6. The bold line is for the deterministic case and the others are for probabilistic case. In here, when the lower limit of the initial processing time is decreased, the time to produce the quantity is decreased.

We can see the changes in time to produce quantity when we change the upper limit of the learning curve slope $l_{b}$ as shown in Figure 3.7. The bold line is for the deterministic case and the others are for probabilistic case. In here, when the upper limit of the learning curve slope is increased, the time to produce the quantity is decreased.

We can see the changes in time to produce quantity when we change the lower limit of 


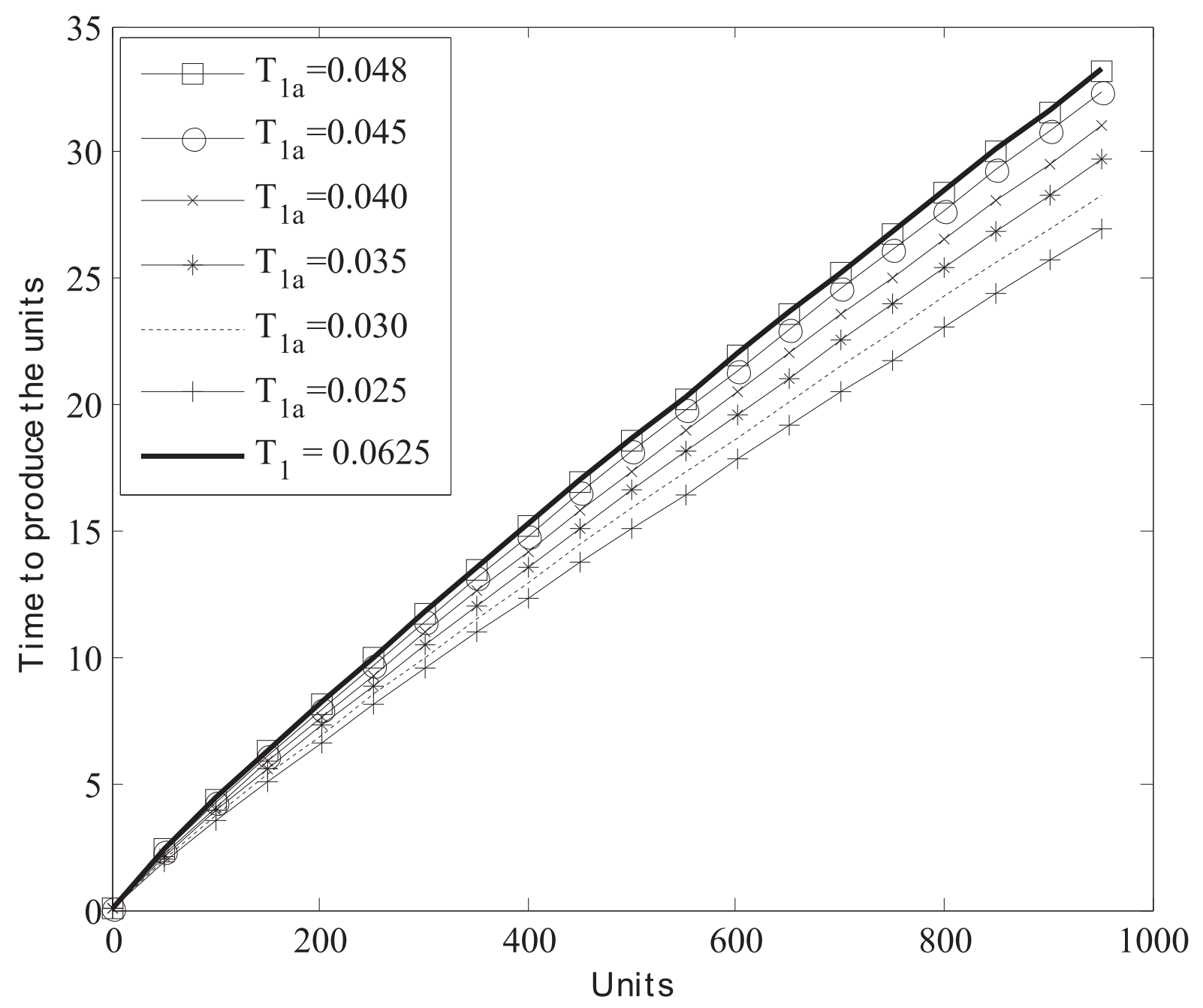

Figure 3.6: Effect on the output when $T_{1 a}$ changes

the initial processing time $l_{a}$ as shown in Figure 3.8. The bold line is for the deterministic case and the others are for probabilistic case. In here, when the lower limit of the learning curve slope is increased, the time to produce the quantity is increased. 


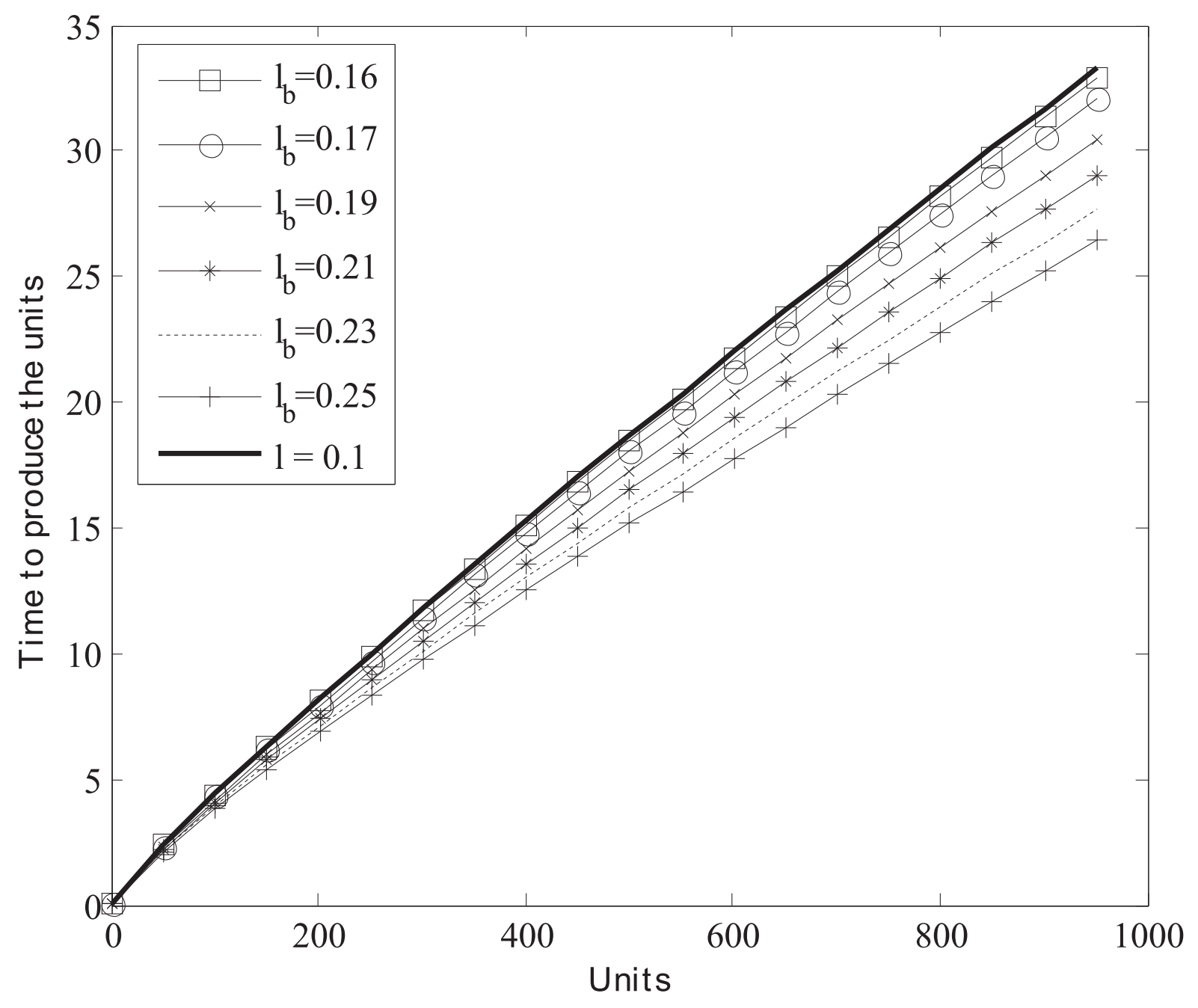

Figure 3.7: Effect on the output when $l_{b}$ changes 


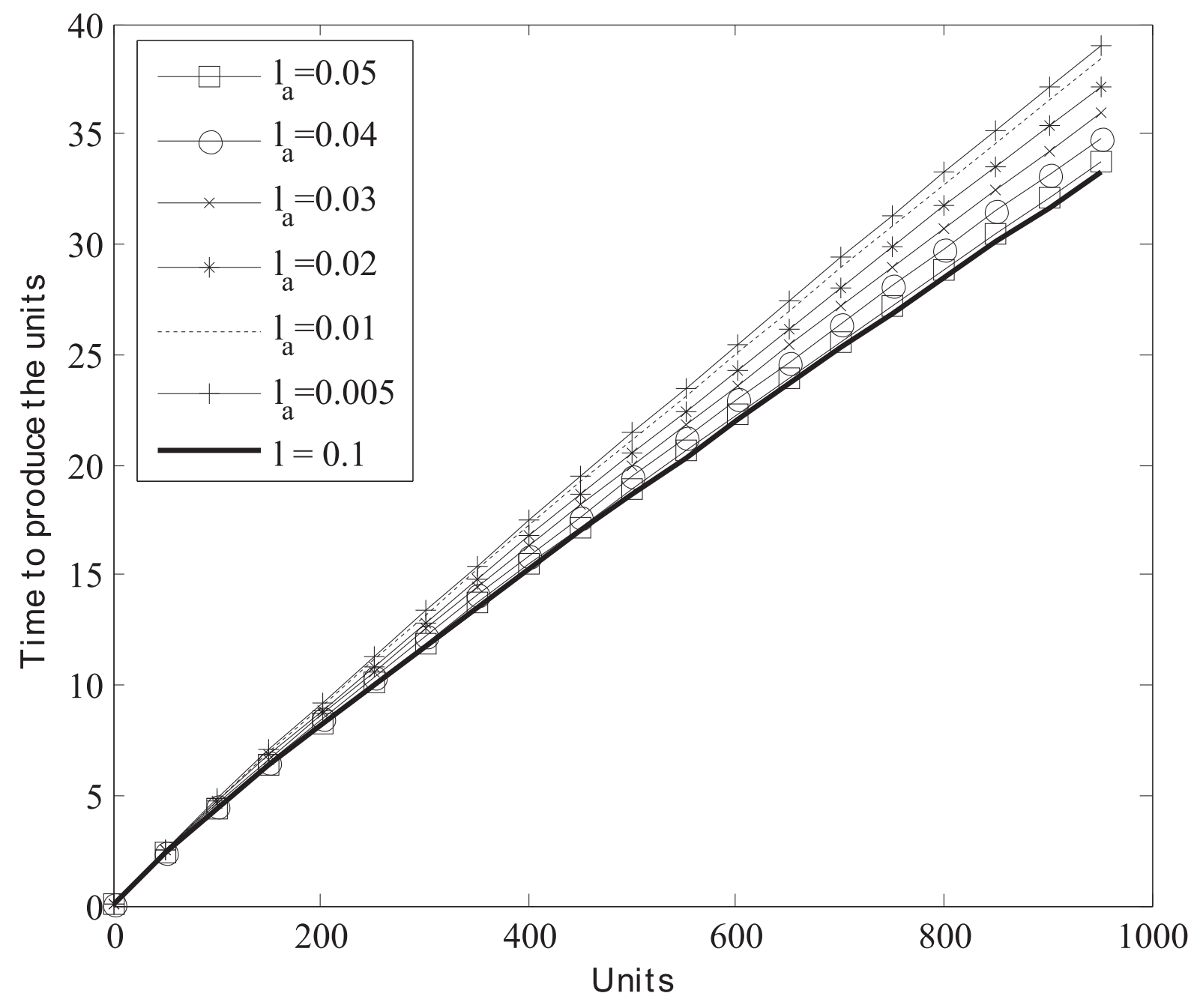

Figure 3.8: Effect on the output when $l_{a}$ changes 


\section{Chapter 4}

\section{Real Options for Cross-Training}

\section{Model}

In this chapter, a real options approach to the valuation of cross-training with three regime product life cycle is introduced. This model can be used to investigate how to cross-train workers who perceive the complexity of certain tasks differently and have learning curve with its individual characteristics. In this chapter, the assumptions are 1) the production system is a serial production system with Kanban release policy, 2) the demand follows three regime product life cycle: growth, maturity, and decay, and 3) each regime is modeled by a geometric Brownian motion.

A heptanomial lattice, having seven branches, is used to model the three-regime product life cycle. The first regime of the product life cycle is the growth regime. Afterwards, it switches stochastically to maturity regime, and then to decay regime. The growth regime represents increasing demand, the maturity regime represents stabilized demand, and the decay regime represents decreasing demand. Each regime is represented by a trinomial lattice and one period is considered as one month. Figure 4.1 shows a single step in a heptanomial lattice. $z$ represents the level of the underlying variable. For example, the branches with nodes $(z, t+1),(z+3, t+1)$, and $(z+6, t+1)$ represent the 
growth regime. The branches with nodes $(z+1, t+1),(z+3, t+1)$, and $(z+5, t+1)$ represent the maturity regime. The branches with nodes $(z+2, t+1),(z+3, t+1)$, and $(z+4, t+1)$ represent the decay regime. To allow the efficient recombination of the branches, the step sizes of all the branches are set to be equal. To maintain the match between the original lattice and the distribution implied by the lattice, the middle branch is added. $\pi_{g, u}, \pi_{g, m}$, and $\pi_{g, d}$ are the branch probabilities of the growth regime of the upper, middle, and lower branches, respectively. $\pi_{m, u}, \pi_{m, m}$, and $\pi_{m, d}$ are the branch probability of the maturity regime of the upper, middle, and lower branches, respectively. Similarly, $\pi_{d, u}, \pi_{d, m}$, and $\pi_{d, d}$ are the branch probability of the decay regime of the upper, middle, and lower branches, respectively. A similar model is presented with two regimes in Bollen (1999).

The step size of regime $w, \hat{\phi}_{w}$, is calculated using mean, $\mu_{w}$, and volatility, $\sigma_{w}$, of the regime $w$ as follows:

$$
\hat{\phi}_{w}=\sqrt{\sigma_{w}^{2} \Delta t+\left(\mu_{w} \Delta t\right)^{2}}
$$

where the $\Delta t$ represents the interval between two layers of the lattice. A small value of $\Delta t$ will make all the condition probabilities of branches positive (Hull 2002).

As Wahab and Lee (2011) explain, $\hat{\phi}_{1}, \hat{\phi}_{2}$, and $\hat{\phi}_{3}$ are the step sizes of each regime: 1.growth, 2.maturity, and 3.decay. First, the step sizes are arranged and re-indexed such that $\phi_{1}<\phi_{2}<\phi_{3} . \hat{\phi}_{1} \neq \phi_{1}$ and $\phi_{1}$ is not the step size of the growth regime. It may belong to any regime but it is the smallest step size. Similarly, $\hat{\phi}_{2}$ and $\hat{\phi}_{3}$ may not be equal to $\phi_{2}$ and $\phi_{3}$, respectively. $\phi_{2}$ and $\phi_{3}$ may not belong to maturity and decay regimes, respectively. Second, they are set for each branch as follows:

$$
\phi=\max \left(\phi_{1}, \frac{\phi_{2}}{2}, \frac{\phi_{3}}{3}\right)
$$

so that $\phi=\frac{\phi_{w}}{w}$ and $\frac{\phi_{w}}{w} \geq \frac{\phi_{\hat{w}}}{\hat{w}}$ for all $\hat{w} \neq w$. $\hat{w}$ also represents a regime. Third, the step 


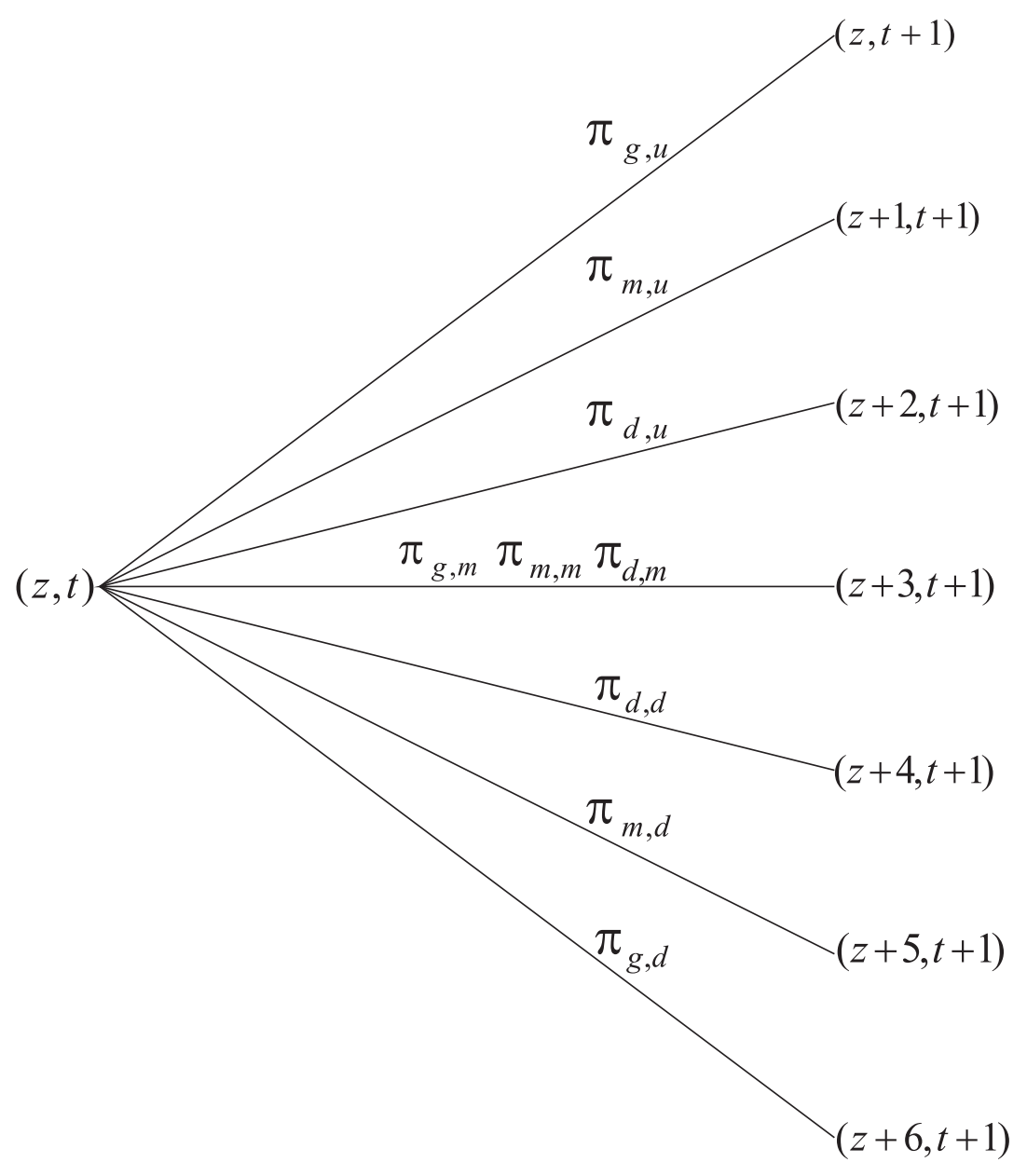

Figure 4.1: Heptanomial lattice

sizes of regimes are adjusted as

$$
\phi_{\hat{w}}= \begin{cases}\phi_{w} & \text { if } \hat{w}=w \\ \hat{w} \frac{\phi_{w}}{w} & \text { if } \hat{w} \neq w\end{cases}
$$

After the step sizes are adjusted, we calculate the branch probabilities of a trinomial lattice for regime $\hat{w}$ as follows:

$$
\begin{aligned}
\pi_{\phi_{\hat{w}}, u} & =\frac{e^{\mu_{\hat{w}} \Delta t}-e^{\left(-\hat{w} \frac{\phi_{w}}{w}\right)}-\pi_{\phi_{\hat{w}}, m}\left(1-e^{\left(-\hat{w} \frac{\phi_{w}}{w}\right)}\right)}{e^{\left(\hat{w} \frac{\phi_{w}}{w}\right)}-e^{\left(-\hat{w} \frac{\phi_{w}}{w}\right)}}, \\
\pi_{\phi_{\hat{w}}, m} & =1-\frac{\phi_{\hat{w}}^{2}}{\left(\hat{w} \frac{\phi_{w}}{w}\right)^{2}}, \\
\pi_{\phi_{\hat{w}}, d} & =1-\pi_{\phi_{\hat{w}}, u}-\pi_{\phi_{\hat{w}}, m},
\end{aligned}
$$




\subsection{Optimization models}

An optimization model is developed to find the optimal cross-training policy. In a serial production system with Kanban release policy, there are $n$ stations with $n$ workers. Product quantity is processed in a series stations, $j(j=1,2, \ldots, n)$. The output of station $j$ is the only input to station $j+1$. The system employs a Kanban release policy where the total inventory of raw material and finished items at each station is kept in a certain level according to the Kanban card set. We assume that if a worker does not work at a secondary station for one period, the worker will completely forget the tasks at the secondary station. The total forgetting is assumed here to justify the cross-training. In this model, the inventory level at a station is maintained at one of $m$ levels.

Index Sets:

$j$ - production stations, $j=1,2, \ldots, n$

$t$ - time period

$k$ - number of workers, $k=1,2, \ldots, n$

Parameters:

$h_{j}$ - holding cost per item per unit time at buffer for station $j$

$C_{j k}$ - cross-training cost at station $j$ for worker $k$

$\tau_{j k}$ - time to produce the first item at station $j$ by worker $k$

$l_{j k}$ - learning slope of worker $k$ for station $j$

$U$ - unit cost per item (including raw material cost and setup cost)

$D_{t}$ - demand in period $t$

$p$ - price per unit of finished product

$b_{j k}$ - labor cost per hour of worker $k$ at station $j$

$\beta_{t}$ - number of labor hours in a period $t$

B - the matrix represents all possible inventory levels

$\mathbf{I}_{\mathbf{t}}$ - a row vector in matrix $\mathbf{B}$ 


\subsubsection{Optimization model with cross-training}

The optimization model, presented in this section, determines the optimal profit when there is a cross-training involved in the production process.

Decision variables:

$I_{j t}$ - inventory at station $j$ at the end of period $t . I_{j t} \in \mathbf{I}_{\mathbf{t}}$ (it is the $j^{t h}$ element of $\mathbf{I}_{\mathbf{t}}$ ) $Q_{j t^{-}}$production quantity at station $j$ during period $t$

$X_{j k t}$ - fraction of the time worker $k$ works at station $j$ in period $t$

$S_{j k t}$ - cross-training status of worker $k$ to station $j$ in period $t$

Maximize the profit in a given period $t$

$\operatorname{Max} V_{C T}=(p-U) Q_{n t}-\sum_{j=1}^{n} h_{j} I_{j t}-\sum_{j=1}^{n} \sum_{k=1}^{n} b_{j k} X_{j k t} \beta_{t}-\sum_{j=1}^{n} \sum_{\substack{k=1 \\ k \neq j}}^{n} C_{j k} S_{j k t}$

Subject to

$$
\begin{array}{lr}
I_{j(t-1)}+Q_{j t}-I_{j t} \leq D_{t}, & j=n \\
I_{j(t-1)}+Q_{j t}-I_{j t}-Q_{(j+1) t}=0, & j=1,2, \ldots, n-1 \\
0 \leq Q_{j t} \leq \sum_{k=1}^{n}\left(\frac{\left(1-l_{j k}\right)\left(\beta_{t}\right)\left(X_{j k t}\right)}{\tau_{j k}}\right)^{1 /\left(1-l_{j k}\right)}, & j=1,2, \ldots, n \\
\sum_{\substack{k=1 \\
k \neq j}}^{n} S_{j k t} \geq 2, & j=1,2, \ldots, n \\
\sum_{\substack{j=1 \\
j \neq k}}^{n} S_{j k t} \geq 2, & \\
S_{j j t}=1, & k=1,2, \ldots, n \\
\sum_{j=1}^{n} X_{j k t} \leq 1, & j=1,2, \ldots, n \\
& k=1,2, \ldots, n
\end{array}
$$




$$
\begin{array}{lr}
X_{j k t} \leq M S_{j k t}, & j=1,2, \ldots, n, k=1,2, \ldots, n \\
0 \leq X_{j k t} \leq 1, & j=1,2, \ldots, n, k=1,2, \ldots, n \\
S_{j k t}=\{0,1\}, & j=1,2, \ldots, n, k=1,2, \ldots, n, k \neq j \\
\mathbf{I}_{\mathbf{t}} \in \mathbf{B}, & j=1,2, \ldots, n \\
I_{j t} \in \mathbf{I}_{\mathbf{t}}, & j=1, n
\end{array}
$$

Constraint (4.8) indicates that the inventory at the last station in $t-1$ period and the quantity produced at period $t$ in the last station is equal to the inventory at the last station in the period $t$ and demand in period $t$. Constraint (4.9) is the balance equation representing the flow at each station. In Constraint (4.10), we calculate the quantity produced at each station by including the effect of learning rate of the workers. Constraints (4.11) and (4.12) are to indicate that at least two workers are cross-trained for each station and each worker is cross-trained for at least two stations in a given period, respectively. Constraint (4.13) indicates that each worker is trained for his or her own station. Constraint (4.14) indicates that the total fraction of the time a worker is assigned to work in all stations in a period should be less than or equal to 1 . Constraint (4.15) is to ensure that a worker is needed to be cross-trained to work on a specific station. That is, if $X_{j k t}>0$, then $S_{j k t}=1$. If $X_{j k t}=0$, then $S_{j k t}=0$. The $M$ is a large integer number. Since the maximum value of $X_{j k t}$ is 1 so, $M=1$. The fraction of the time assigned to a worker, $X_{j k t}$, is between 0 and 1 (Constraint (4.16)). Constraint (4.17) indicates that the cross-training status is either 1 or 0 . Constraint (4.18) indicates that the buffer inventory for each station in a given period is represented by one of the rows of matrix $\mathbf{B}$. The matrix $\mathbf{B}$ has $n^{m}$ rows. The profit is maximized by searching over all possible $n^{m}$ rows. Constraint (4.19) indicates that $I_{j t}$ is an element of the vector of inventory level $\mathbf{I}_{\mathbf{t}}$; that is $I_{j t}$ is the inventory level at station $j$. 


\subsubsection{Optimization model without cross-training}

The optimization model, presented in this section, calculates the optimal profit without cross-training. In this model, each worker works on his or her primary station only. The following optimization model is created without any cross-training aspects.

Decision variables:

$I_{j t}$ - inventory at station $j$ at the end of period $t$

$Q_{j t^{-}}$production quantity at station $j$ during period $t$

Maximize the profit in a given period $t$

$$
\operatorname{Max} V_{N C T}=(p-U) Q_{n t}-\sum_{j=1}^{n} h_{j} I_{j t}-\sum_{\substack{j=1 \\ k=j}}^{n} b_{j k} \beta_{t}
$$

Subject to

$$
\begin{array}{lr}
I_{j(t-1)}+Q_{j t}-I_{j t} \leq D_{t}, & \forall j=n \\
I_{j(t-1)}+Q_{j t}-I_{j t}-Q_{(j+1) t}=0, & \forall j=1,2, \ldots, n-1 \\
0 \leq Q_{j t} \leq \sum_{k=1}^{n}\left(\frac{\left(1-l_{j k}\right)\left(\beta_{t}\right)}{\tau_{j k}}\right)^{1 /\left(1-l_{j k}\right)}, & \forall j=1,2, \ldots, n \\
\mathbf{I}_{\mathbf{t}} \in \mathbf{B}, & j=1,2, \ldots, n \\
I_{j t} \in \mathbf{I}_{\mathbf{t}}, &
\end{array}
$$

Constraint (4.21) indicates that the inventory at the last station in $t-1$ period and the quantity produced at period $t$ in the last station is equal to the inventory at the last station in the period $t$ and demand in period $t$. The constraint (4.22) is the balance equation of the inventory level and production quantity. In Constraint (4.23), we calculate the quantity produced at each station by including the effect of learning rate of the workers. Constraint (4.24) indicates that the inventory stored at each station is one of the row vector of matrix $\mathbf{B}$. The matrix $\mathbf{B}$ has $n^{m}$ rows. The profit is maximized 
by searching over all the $n^{m}$ rows. Constraint (4.25) indicates that $I_{j t}$ is an element of the vector of inventory level $\mathbf{I}_{\mathbf{t}}$; that is $I_{j t}$ is the inventory level at station $j$.

\subsection{Dynamic programming}

After we find the optimal profit at each node of the demand developed by the lattice approach, the backward dynamic program is used to find the net present value. For the terminal node, the net present value is just the cash flow and it is calculated as follows:

$$
V\left(z, \mathbf{I}_{t}, T\right)=\max \left[V_{C T}\left(z, \mathbf{I}_{t}\right), V_{N C T}\left(z, \mathbf{I}_{t}\right)\right], \quad \text { when } t=T \quad \forall z
$$

where $z$ represents the level of the underlying variable in a lattice. $V_{C T}\left(z, \mathbf{I}_{t}\right)$ is the maximum profit computed with cross-training whereas $V_{N C T}\left(z, \mathbf{I}_{t}\right)$ is the maximum profit computed without cross-training.

For the intermediate nodes, the net present value is present value cash flow plus the discounted expected value from the next time period. The net present value of each node is computed using inventory level of prior node and the demand. The maximum profit for each level of inventory is calculated as follows:

$$
P V\left(z, \hat{w}, \mathbf{I}_{t}, t\right)=\max _{\mathbf{B}_{t}}\left[V\left(z, \mathbf{I}_{t}, t\right), E V\left(z, \hat{w}, \mathbf{I}_{t+1}, t\right)-H\left(\mathbf{I}_{t}, \mathbf{I}_{t+1}\right)\right], \forall \hat{w} \in\{g, m, d\}, t \neq T
$$

where $P V\left(z, \hat{w}, \mathbf{I}_{t}, t\right)$ is the present value in regime $\hat{w}$ at time $t$ and current inventory level $\mathbf{I}_{t} . H\left(\mathbf{I}_{t}, \mathbf{I}_{t+1}\right)$ is the holding cost. $V\left(z, \mathbf{I}_{t}, t\right)$ is the maximum profit computed using the optimization model.

$E V\left(z, \hat{w}, \mathbf{I}_{t}, t\right)$ is the discounted expected value. It differs from regime to regime because each regime is constructed by different set of branches. The discounted expected 
value for the growth regime is calculated as follows:

$$
\begin{aligned}
E V\left(z, g, \mathbf{I}_{t}, t\right) & =e^{-r \Delta t}\left[( 1 - p ( t ) ) \left(\pi_{g, u} P V\left(z, g, \mathbf{I}_{t}, t+1\right)\right.\right. \\
& \left.+\pi_{g, m} P V\left(z+3, g, \mathbf{I}_{t}, t+1\right)+\pi_{g, d} P V\left(z+6, g, \mathbf{I}_{t}, t+1\right)\right) \\
& +p(t)\left(\pi_{m, u} P V\left(z+1, m, \mathbf{I}_{t}, t+1\right)+\pi_{m, m} P V\left(z+3, m, \mathbf{I}_{t}, t+1\right)\right. \\
& \left.\left.+\pi_{m, d} P V\left(z+5, m, \mathbf{I}_{t}, t+1\right)\right)\right]
\end{aligned}
$$

where $g$ represents the growth regime and $m$ represents the maturity regime. $p(t)$ is the probability of switching from the growth regime to the maturity regime. $\pi_{g, u}, \pi_{g, m}$, and $\pi_{g, d}$ are the conditional branch probabilities of the growth regime of the upper, middle, and lower branches, respectively. Similarly, $\pi_{m, u}, \pi_{m, m}$, and $\pi_{m, d}$ are the conditional branch probabilities of the maturity regime of the upper, middle, and lower branches, respectively.

The discounted expected value for the maturity regime is calculated as follows.

$$
\begin{aligned}
E V\left(z, m, \mathbf{I}_{t}, t\right) & =e^{-r \Delta t}\left[( 1 - q ( t ) ) \left(\pi_{m, u} P V\left(z+1, m, \mathbf{I}_{t}, t+1\right)\right.\right. \\
& \left.+\pi_{m, m} P V\left(z+3, m, \mathbf{I}_{t}, t+1\right)+\pi_{m, d} P V\left(z+5, m, \mathbf{I}_{t}, t+1\right)\right) \\
& +q(t)\left(\pi_{d, u} P V\left(z+2, d, \mathbf{I}_{t}, t+1\right)+\pi_{d, m} P V\left(z+3, d, \mathbf{I}_{t}, t+1\right)\right. \\
& \left.\left.+\pi_{d, d} P V\left(z+4, d, \mathbf{I}_{t}, t+1\right)\right)\right],
\end{aligned}
$$

where $d$ represents the decay regime. $q(t)$ is the probability of switching from the maturity regime to the decay regime. $\pi_{d, u}, \pi_{d, m}$, and $\pi_{d, d}$ are the conditional branch probabilities of the decay regime of the upper, middle, and lower branches, respectively.

The discounted expected value for the decay regime is calculated as follows:

$$
\begin{aligned}
E V\left(z, d, I_{t}, t\right) & =e^{-r \Delta t}\left[\pi_{d, u} P V\left(z+2, d, \mathbf{I}_{t}, t+1\right)\right. \\
& \left.+\pi_{d, m} P V\left(z+3, d, \mathbf{I}_{t}, t+1\right)+\pi_{d, d} P V\left(z+4, d, \mathbf{I}_{t}, t+1\right)\right] .
\end{aligned}
$$

In Equation (4.27), $H\left(\mathbf{I}_{t}, \mathbf{I}_{t+1}\right)$ is the holding cost. Holding cost is involved when the inventory level changes from $\mathbf{I}_{t}$ and $\mathbf{I}_{t+1}$. It will be deducted from the expected value to 
get the net present profit. The holding cost is calculated as follows:

$$
H\left(\mathbf{I}_{t}, \mathbf{I}_{t+1}\right)=\sum_{j=1}^{4} h_{j}\left(I_{j(t+1)}-I_{j t}\right)
$$

where $h_{j}$ is the inventory carrying cost per unit per period at station $j$.

\subsection{Numerical analysis}

Let us assume the total life cycle of a product is 5-year period, which is discretized monthly. So, there are 60 periods in the total product life cycle $(t l)$. The switch from the growth regime to the maturity regime in the product life cycle is modeled as a cumulative normal distribution with probability with a mean $\left(\mu_{g m}\right)$ of 3 years and a standard deviation of 3 months. The switch from the maturity regime to the decay regime is modeled with probability with a mean $\left(\mu_{m d}\right)$ of 4 year and a standard deviation of 3 months. The initial demand is 180 . The risk free rate of interest is $5 \%$. Unit cost per unit (including raw material and setup cost) is 2 . The price per unit is $\$ 25.50$. There are 4 stations and 4 workers in the production line. The profit is maximized by searching over four levels of inventory $(4,8,12$ and 16$)$, which leads to $4^{4}=256$ rows. We use Kanban WIP system because we have a constant inventory level at each station in a particular period. We are using optimization model to calculate the maximum profit for each inventory level. Number of hours in a period is 300 hours.

Table 4.1 shows the annual risk adjusted mean and volatility that is used for each regime of product life cycle. Table 4.2 shows the holding costs involved in each station and there are 4 stations. Table 4.3 shows the learning slope of each worker at each station. Table 4.4 shows the time to produce the first unit for each worker at each station. Table 4.5 shows the labor cost for each worker at each station. Table 4.6 shows the cross-training cost for each worker at each station.

The single regime process parameters were carefully selected so it can be compared with three regime product life cycle. The volatility $\left(\sigma_{b}\right)$ is calculated so the binomial 
Table 4.1: Annual risk adjusted mean and volatility for each regime of product life cycle

\begin{tabular}{|l|c|c|}
\hline & annual risk adjusted mean $(\mu)$ & volatility $(\sigma)$ \\
\hline growth regime & $25 \%$ & $30 \%$ \\
maturity regime & $0 \%$ & $15 \%$ \\
decay regime & $-25 \%$ & $5 \%$ \\
\hline
\end{tabular}

Table 4.2: Holding costs involved in each station

\begin{tabular}{|l|c|c|c|c|}
\hline & station 1 & station 2 & station 3 & station 4 \\
\hline holding cost & $\$ 0.10$ & $\$ 0.20$ & $\$ 0.30$ & $\$ 0.40$ \\
\hline
\end{tabular}

Table 4.3: Learning slope of each worker at each station

\begin{tabular}{|l|c|c|c|c|}
\hline & worker 1 & worker 2 & worker 3 & worker 4 \\
\hline station 1 & 0.20 & 0.20 & 0.30 & 0.30 \\
station 2 & 0.10 & 0.30 & 0.20 & 0.10 \\
station 3 & 0.30 & 0.10 & 0.10 & 0.20 \\
station 4 & 0.20 & 0.10 & 0.10 & 0.30 \\
\hline
\end{tabular}

Table 4.4: Time to produce first unit for each worker at each station

\begin{tabular}{|l|c|c|c|c|}
\hline & worker 1 & worker 2 & worker 3 & worker 4 \\
\hline station 1 & 0.01 & 0.01 & 0.02 & 0.01 \\
station 2 & 0.02 & 0.01 & 0.02 & 0.01 \\
station 3 & 0.01 & 0.02 & 0.02 & 0.01 \\
station 4 & 0.01 & 0.01 & 0.02 & 0.02 \\
\hline
\end{tabular}

lattice is covered the entire three regime product life cycle. $\sigma_{b}$ is selected for geometric Brownian motion by a weighted average of the three regimes' volatilities.

$$
\sigma_{b}=\sqrt{\frac{\mu_{g m}}{t l} \sigma_{g}^{2}+\frac{\mu_{m d}-\mu_{g m}}{t l} \sigma_{m}^{2}+\frac{t l-\mu_{m d}}{t l} \sigma_{d}^{2}}
$$

The mean value for single regime product life cycle is also carefully selected to compare 
Table 4.5: Labor cost for each worker

\begin{tabular}{|l|c|c|c|c|}
\hline & worker 1 & worker 2 & worker 3 & worker 4 \\
\hline station 1 & $\$ 10$ & $\$ 11$ & $\$ 10$ & $\$ 12$ \\
station 2 & $\$ 10$ & $\$ 10$ & $\$ 11$ & $\$ 12$ \\
station 3 & $\$ 10$ & $\$ 10$ & $\$ 10$ & $\$ 12$ \\
station 4 & $\$ 10$ & $\$ 11$ & $\$ 12$ & $\$ 10$ \\
\hline
\end{tabular}

Table 4.6: Cross-training cost for each worker

\begin{tabular}{|c|c|c|c|c|}
\hline & worker 1 & worker 2 & worker 3 & worker 4 \\
\hline station 1 & $\$ 0.00$ & $\$ 100.50$ & $\$ 150.00$ & $\$ 150.50$ \\
station 2 & $\$ 200.00$ & $\$ 0.00$ & $\$ 100.00$ & $\$ 110.50$ \\
station 3 & $\$ 100.00$ & $\$ 150.50$ & $\$ 0.00$ & $\$ 100.50$ \\
station 4 & $\$ 170.00$ & $\$ 110.50$ & $\$ 150.00$ & $\$ 0.00$ \\
\hline
\end{tabular}

with three regime product life cycle. It is selected using trial and error procedure. The mean value, which gives the same expected discount profit for both with product life cycle and without product life cycle models, is selected.

Figure 4.2 shows that when we calculate the profit and the cross-training status for model without product life cycle, we can see that the upper part of lattice gives the maximum profit with cross-training and the lower part gives the maximum profit without cross-training. The figure shows that the cross-training is helpful only in the last period. If we increase the number of periods, the cross-training will help more and we may see it when the time approaches the end of the period. When the time approaches zero, crosstraining is not helpful. It shows that when the demand is higher, the cross-training helps to maximize the profit. If the demand is lower, then there is no need for cross-training. The cross-training status is same for model with product life cycle. Figure 4.3 shows the profit and the cross-training status for model with product life cycle.

The change in profit when we alter the volatility of growth regime $\left(\sigma_{g}\right)$ is shown in 


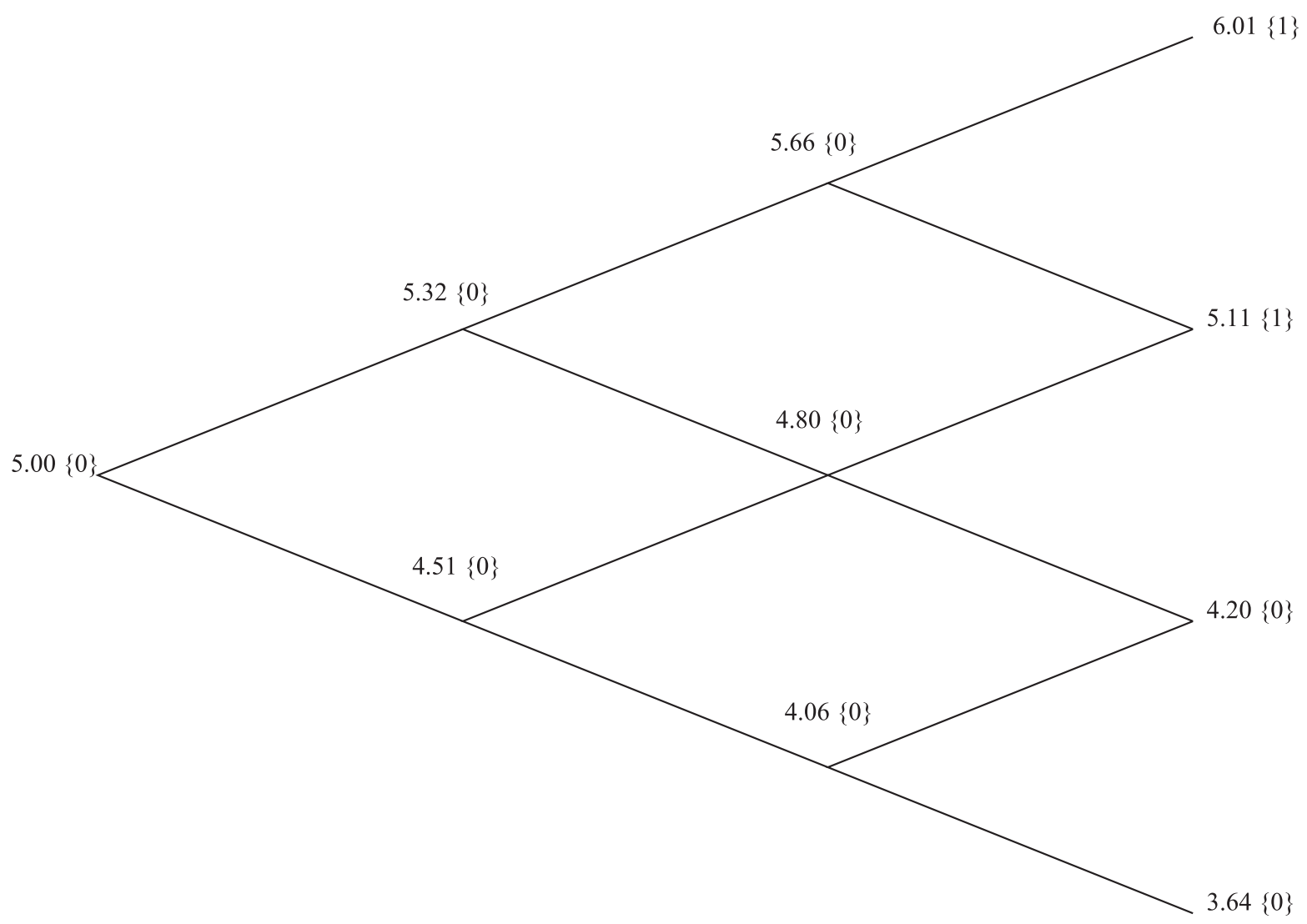

Figure 4.2: Cross-training status for three periods of binomial lattice

Figure 4.4. The single regime process volatility $\left(\sigma_{b}\right)$ is calculated by a weighted average of the three regime's volatilities so it can be compared with three regime product life cycle. Therefore, it is also shown in Figure 4.4 to compare. The profit of the model without product life cycle is way higher than the results from the model with product life cycle. It shows how the product life cycle affects the profit. When there is no product life cycle, the profit is not calculated accurately. Also, the profit increases significantly when the volatility of growth regime $\left(\sigma_{g}\right)$ is increased because the larger volatility is, the greater is the probability that demand will grow significantly more.

The change in profit when we alter the volatility of maturity regime $\left(\sigma_{m}\right)$ is shown in Figure 4.5. The profit of the model without product life cycle is way higher than the results of the model with product life cycle. It shows how the product life cycle affects the 


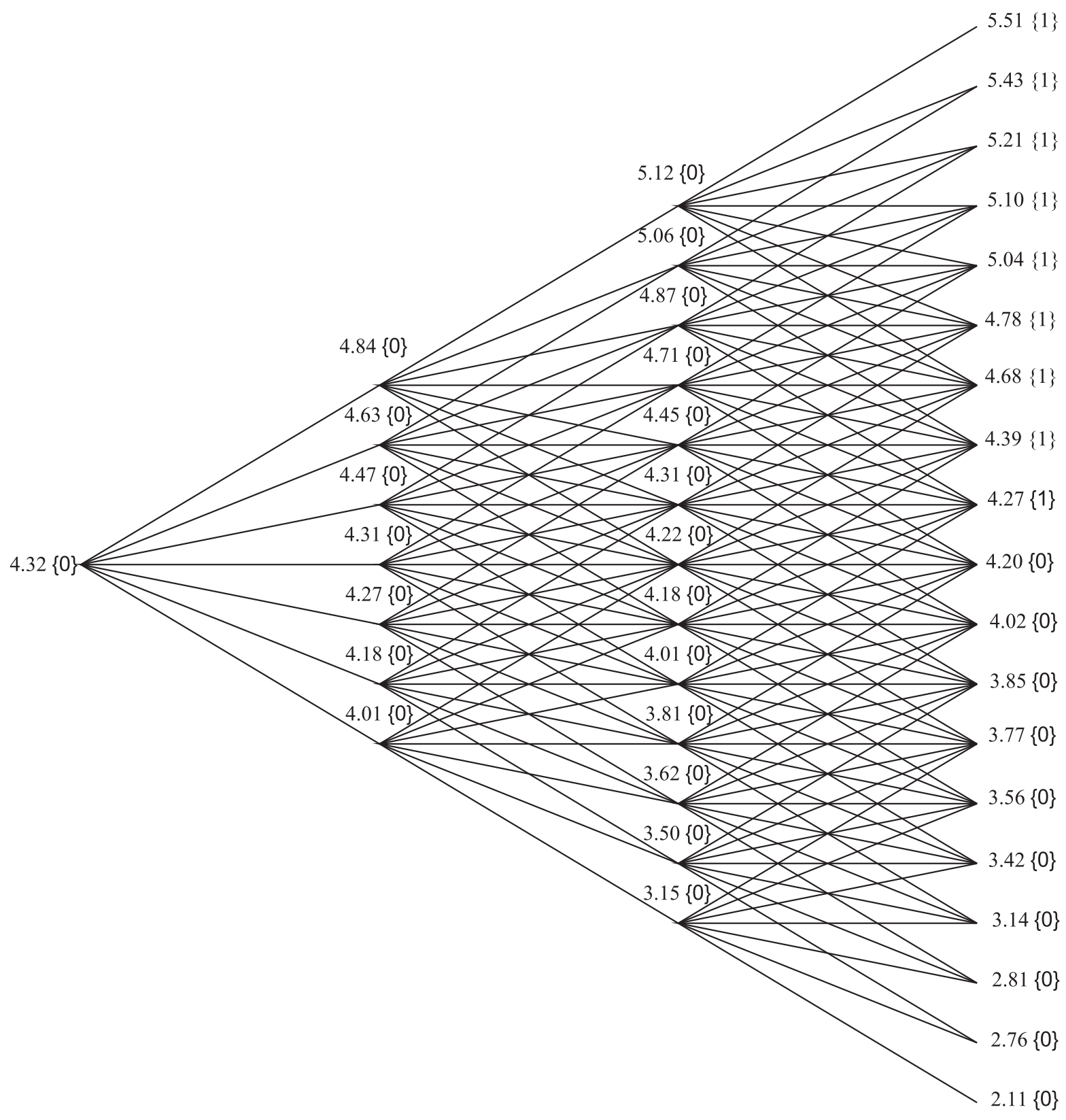

Figure 4.3: Cross-training status for three periods of heptanomial lattice

profit. When there is no product life cycle, the profit is not calculated accurately. Also, the profit does not change much when the volatility of maturity regime $\left(\sigma_{m}\right)$ changes because there is no change in demand in maturity regime.

The change in profit when we change the volatility of decay regime $\left(\sigma_{d}\right)$ is shown in Figure 4.6. The profit of the model without product life cycle is way higher than the 


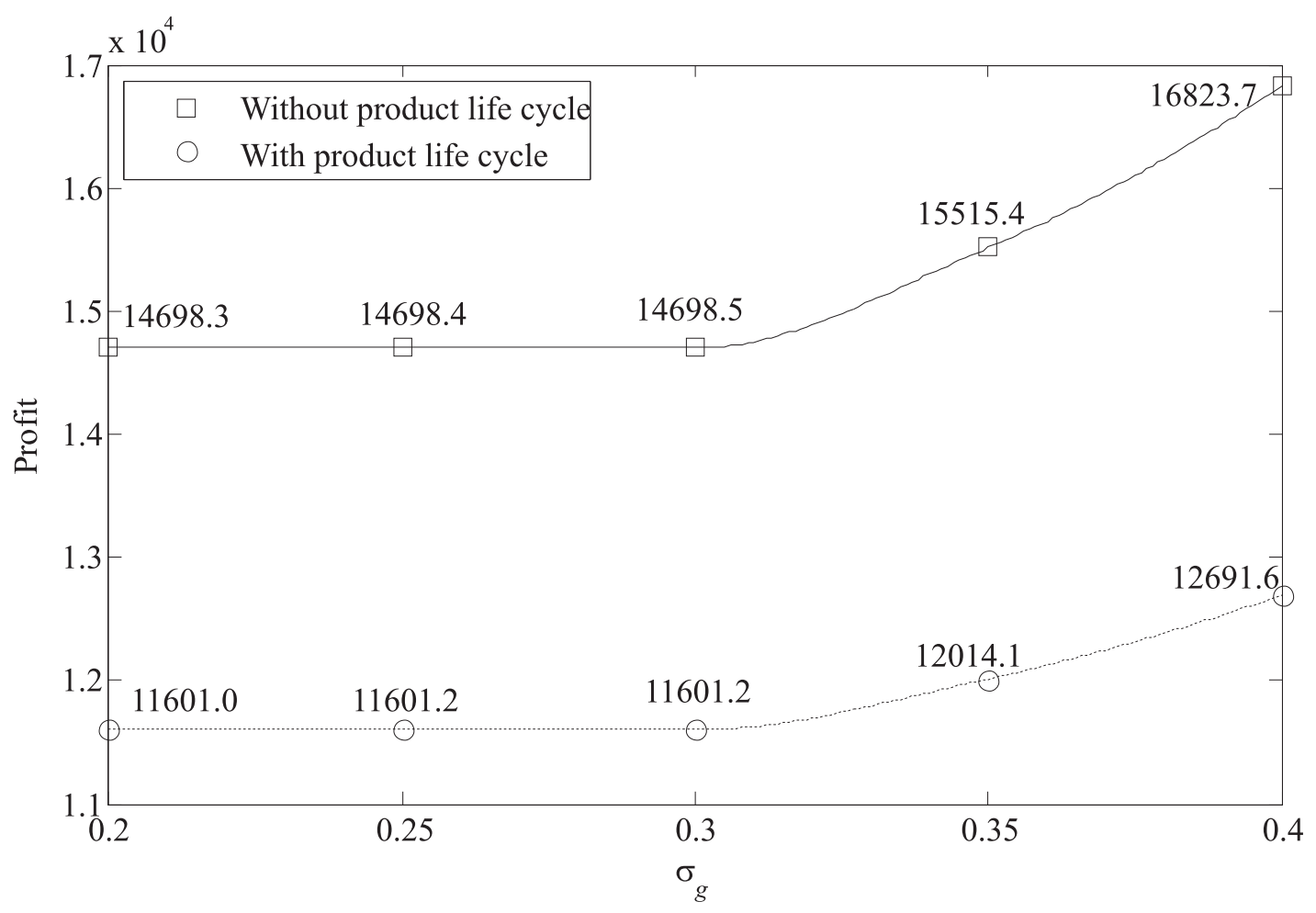

Figure 4.4: Change in profit vs. volatility of growth regime $\left(\sigma_{g}\right)$

results of the model with product life cycle. It shows how the product life cycle affects the profit. We saw the same pattern earlier in the volatilities of growth and maturity regimes. The profit increases when the volatility of decay regime $\left(\sigma_{d}\right)$ is increased because the larger volatility is, the demand significantly less. So the increase in the profit is very low.

The change in profit when we change the annual risk adjusted mean of growth regime $\left(\mu_{g}\right)$ is shown in Figure 4.7. Here also we can see that the result of the model without product life cycle is higher than the results of the model with product life cycle. Also, the profit increases significantly when the annual risk adjusted mean of growth regime $\left(\mu_{g}\right)$ increases. It shows that profit is higher the higher the mean is, since this implies that the life cycle will likely remain in growth for a longer period of time. The profit that was calculated from the model without product life cycle is increased faster than the profit from the model with product life cycle. 


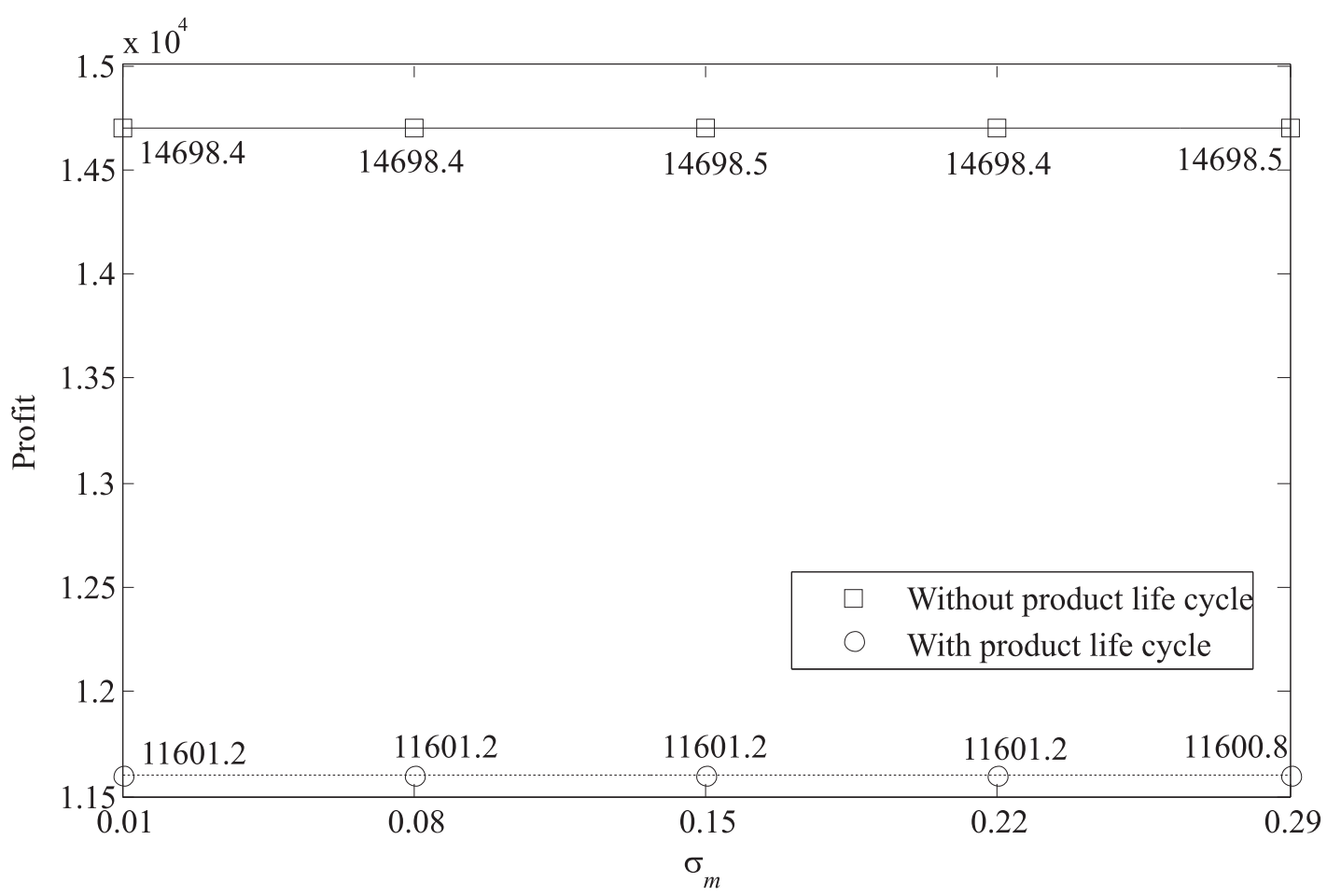

Figure 4.5: Change in profit vs. volatility of maturity regime $\left(\sigma_{m}\right)$

The change in profit when we increase the years of life cycle is shown in Figure 4.8. In this result also, we can see how the product life cycle affects. Without product life cycle, the results will not be accurate. Also, the profit increases significantly when the years of life cycle increases. It shows that profit is higher the longer the year is, since this implies that the life cycle will likely remain in growth for a longer period of time. The profit that was calculated from the model with product life cycle is increased faster than the profit from the model without product life cycle.

The change in profit, when the number of stations cross-trained is altered, is shown in Figure 4.9. The profit changes significantly at the beginning but after two stations, the result is saturated. It happened because after a certain number of stations cross-trained, the effect on the profit is minimal. It is because the worth of the cross-trained workesr is lesser than the cross-training cost. 


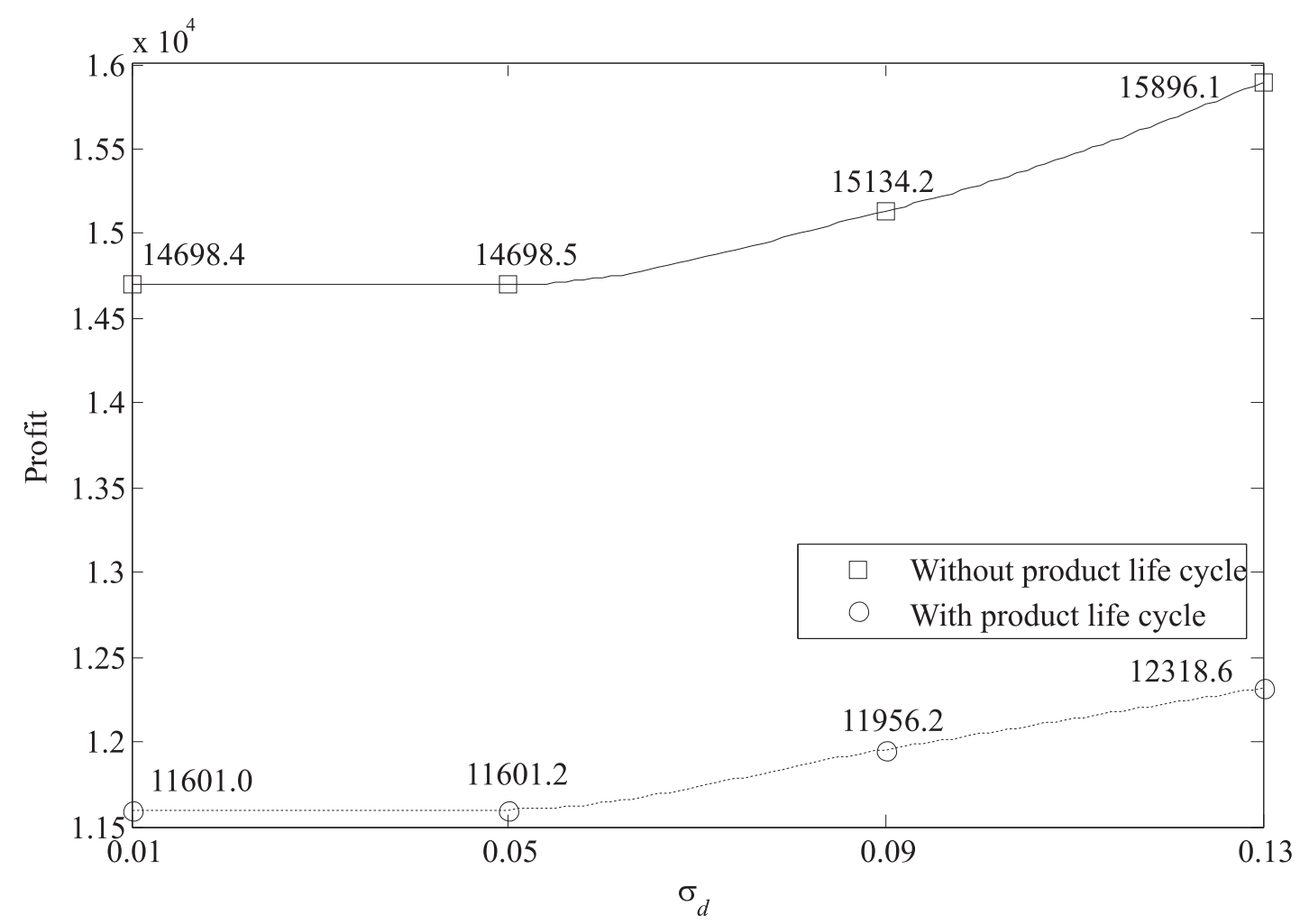

Figure 4.6: Change in profit vs. volatility of decay regime $\left(\sigma_{d}\right)$

The change in profit, when the number of workers cross-trained is altered, is shown in Figure 4.10. The profit changes significantly at the beginning but after two workers, there is not much change in profit. It happened because after a certain number of workers cross-trained, the effect on the profit is minimal. It is because the worth of the crosstrained stations is lesser than the cross-training cost. 


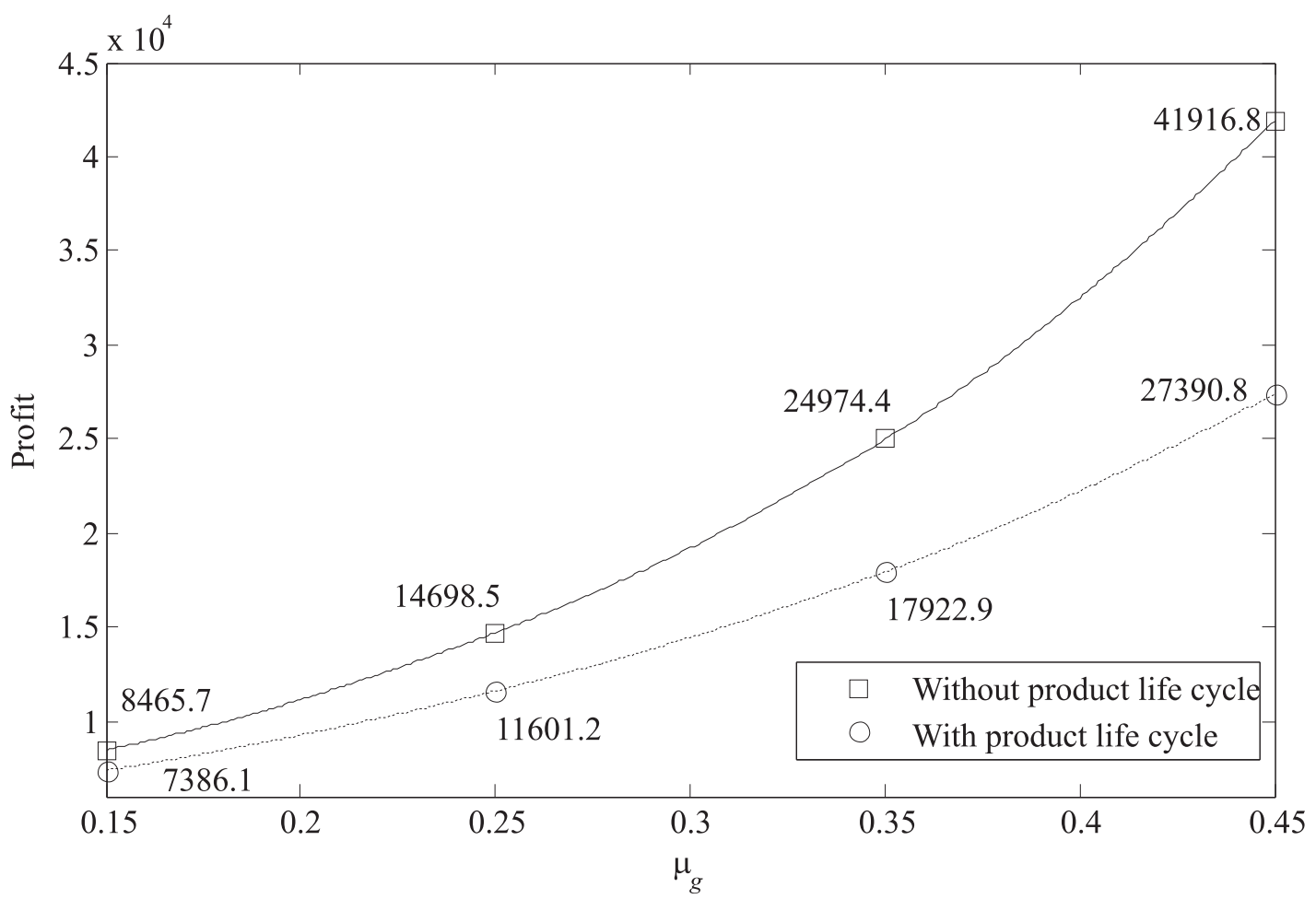

Figure 4.7: Change in profit vs. annual risk adjusted mean $\left(\mu_{g}\right)$

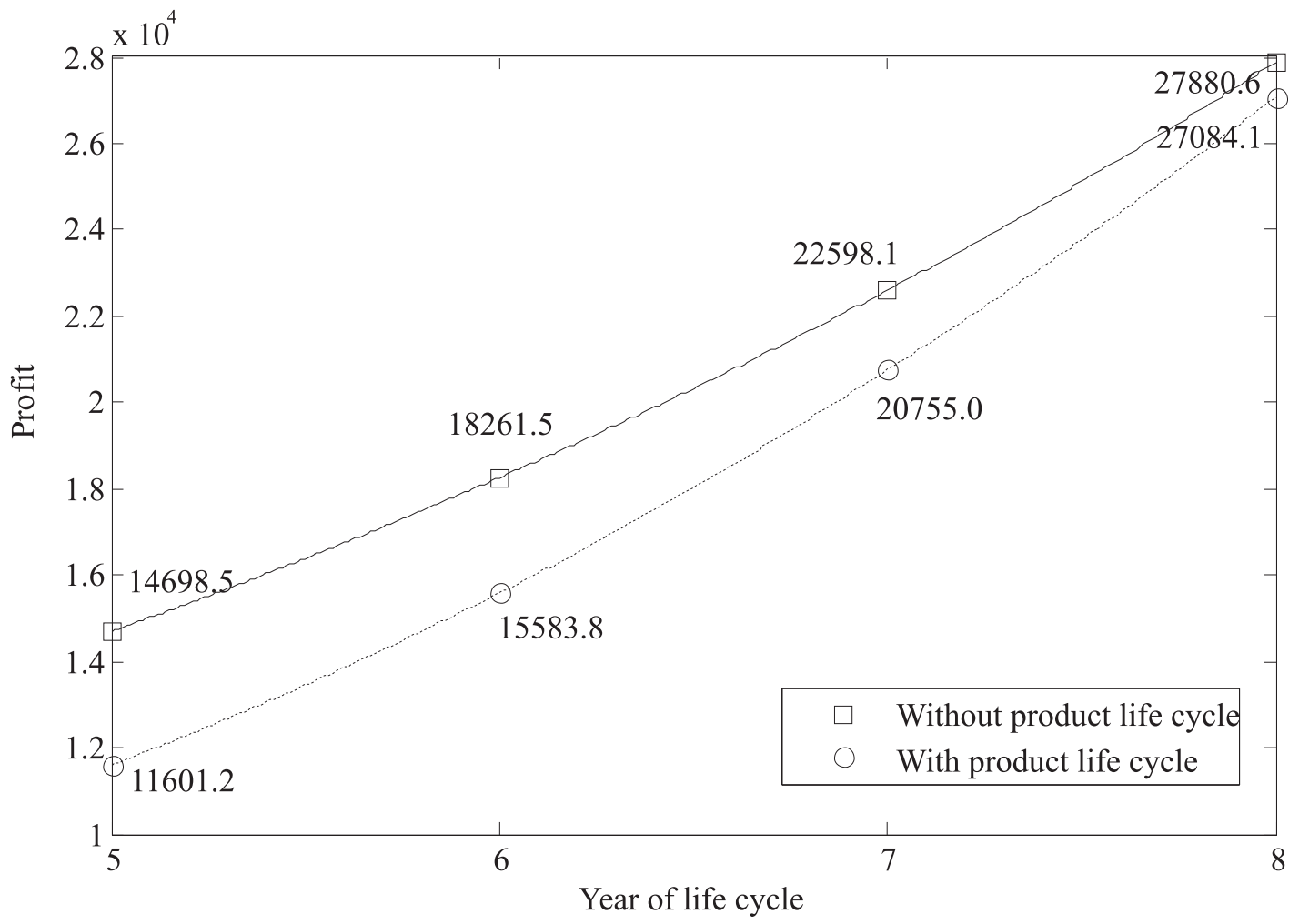

Figure 4.8: Change in profit vs. years of lifecycle 


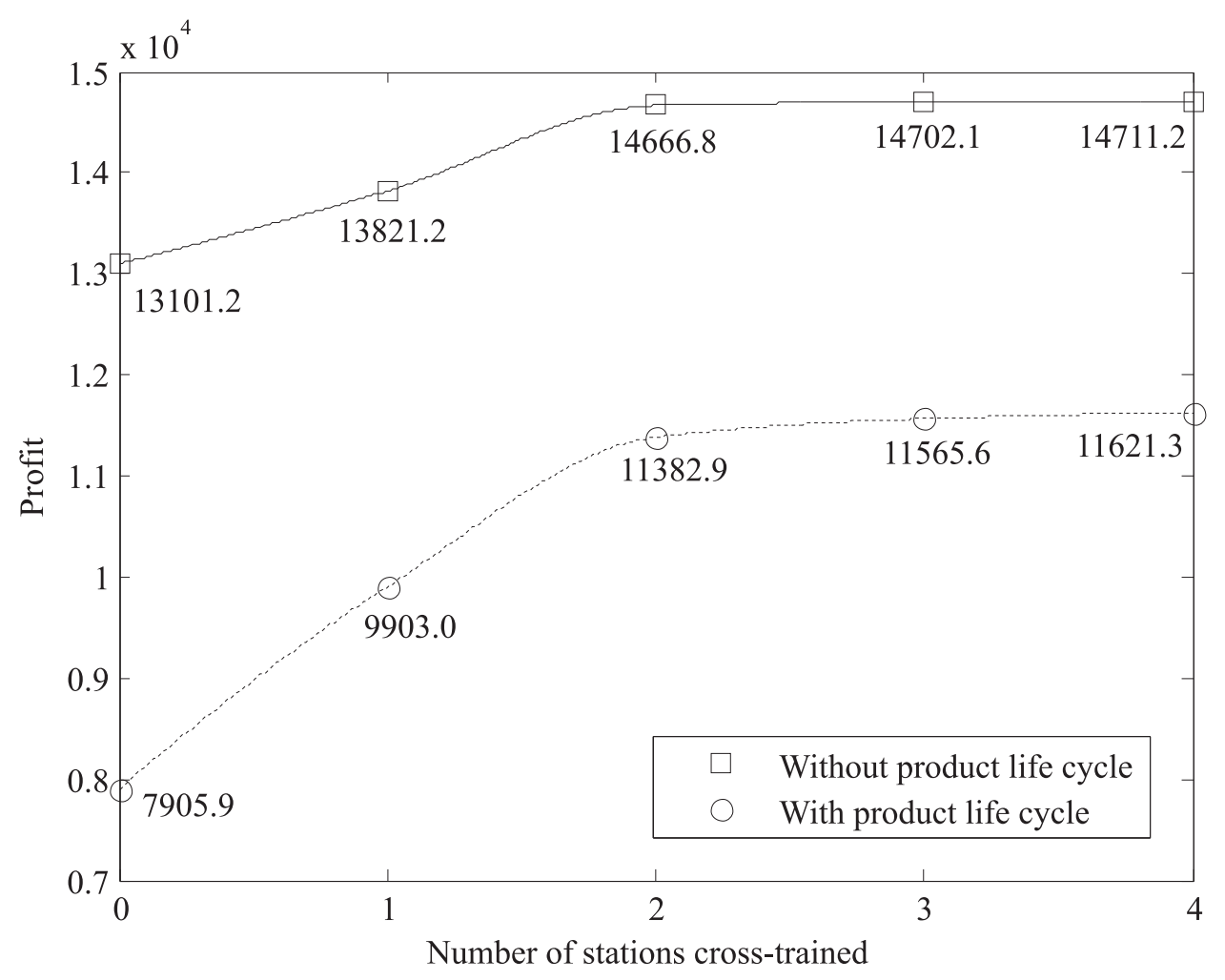

Figure 4.9: Change in profit vs. number of stations cross-trained 


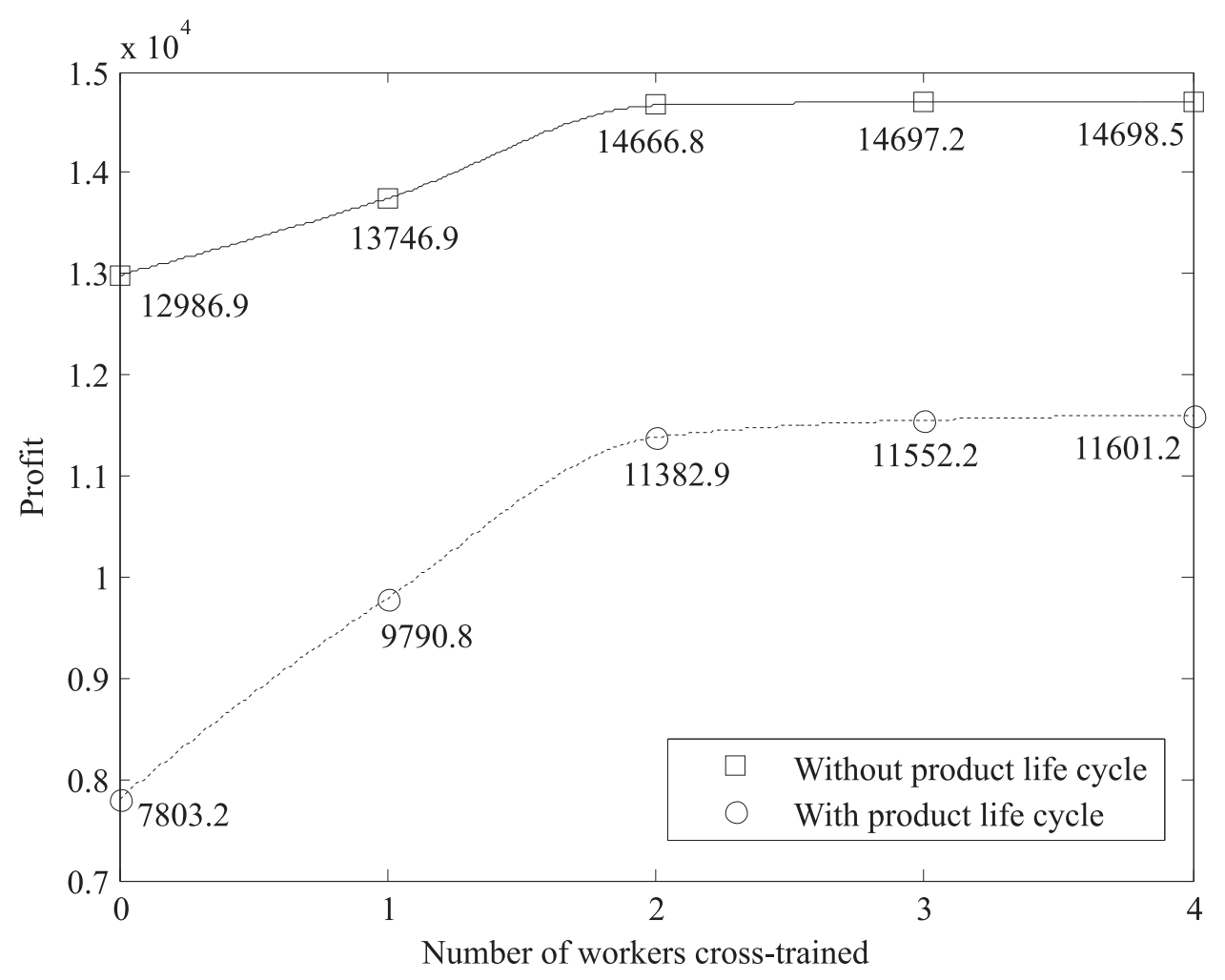

Figure 4.10: Change in profit vs. number of workers cross-trained 


\section{Chapter 5}

\section{Conclusion}

In this study, we have developed two models: (i) a probabilistic learning curve approach to the production lot size problem to determine the economic manufactured quantity (EMQ); (ii) a real options approach to the valuation of cross-training with product life cycle. We modify the learning curve equation for the probabilistic learning curve slope and the time required to produce the first unit and find the unit cost function. For simplicity, we use uniform distribution functions for both the learning rate and the time required to produce the first unit. The results obtained from probabilistic learning curve slope and time to produce the first unit are significantly different from those with the constant learning curve slope and time to produce the first unit. When we had the deterministic case, we found the optimum quantity of time to forget in 300 days is 248 in cycle 1 but in probabilistic case, it was 465. The amount of time required to produce 248 units is 9.92 days and to produce 465 units in 17.65 days. The total output, when no interruption occurred was 560 units and the forgetting slope was 0.1442 . For the probabilistic case, the total output, when no interruption occurred was 1126 units and the forgetting slope was 0.1913 .

All the workers do not have the same learning rate or do not finish the work in the same time frame. Even one worker always does not have the ability finish one product 
is same time frame over and over again. When we give probabilistic learning rate and time to do the units, it makes the process more realistic. The results significantly differ when we use probabilistic parameters to model the learning curve than the results with constants parameters. Therefore, the probabilistic results are more realistic one.

For real options approach to the valuation of cross-training with product life cycle, we consider three regime product life cycle: growth, maturity, and decay. The demand follows the stochastic process modeled using a Brownian motion. Because there are three regimes in the product life cycle, we use heptanomial lattice to represent it. We compare the results of model with product life cycle and without product life cycle. The parameters for without product life cycle are carefully selected so that geometric Brownian motion covers the entire three regime product life cycle.

When we calculate the profit and the cross-training status for model with product life cycle, we can see that the upper part of lattice gives the maximum profit with crosstraining and the lower part gives the maximum profit without cross-training. It shows that when the demand is higher and the profit is higher, the cross-training helps to profit more. If the demand is lower and the profit is lower, then there is no need for crosstraining. The results from model with product life cycle and model without product life cycle is differ significantly. It shows how the product life cycle affects the profit. The profit cannot be calculated accurately without product life cycle. When we change the volatility for growth regime $\left(\sigma_{g}\right)$ and the volatility for decay regime $\left(\sigma_{d}\right)$, the profit changes significantly, but the volatility for maturity regime $\left(\sigma_{m}\right)$ does not change the profit too much. However, when we change the annual risk adjusted mean for growth regime $\left(\mu_{g}\right)$, the profit changes significantly. The number of years of the product life cycle also affects the results significantly. The profit increases when the number of stations or number of workers increases. After a certain number of stations or workers cross-trained, there is not much change in profit, it saturated. The results are calculated using the actual dollar values. This will help the managers to make decisions easily and effectively. 
There are some limitations related to the models in the thesis. One being that we did not include the probabilistic learning curve in the optimization model to calculate the quantity produced. It will discretize the lattice with more nodes making the numerical computation harder.

When the time to produce the quantity is calculated, we are using uniform distribution. Even with the uniform distribution, we needed to use Matlab to find the numerical results. If we use any other distribution, other than uniform distribution, the complexity of the equation will be very hard. This is another limitation related to the model.

Further limitation in our model is regarding the number of stations and workers, and inventory levels. We had four stations, four workers, and four inventory levels. It will give a matrix with $4^{4}=256$ rows. This means when we calculate profit at each node of the heptanomial lattice, we will have 256 different results at each node. If we increase the number of stations, number of workers, and inventory levels, it will make the computation very complex and will take long time to run the program to find the results.

For future work, expand the model to include these above limitations and write programs to handle complex computations. Also, learning curve equation can be modified to find unit cost for production analysis with back orders. 


\section{Appendix A}

Derivation for Equation 3.20 is given below:

$$
\begin{gathered}
t^{s}\left(q_{i}\right)=\int_{\alpha_{i}}^{q_{i}+\alpha_{i}} \int_{l_{a}}^{l_{b}} \int_{T_{1 a}}^{T_{1 b}} T_{1} x^{-l} \frac{1}{\left(T_{1 b}-T_{1 a}\right)} \frac{1}{\left(l_{b}-l_{a}\right)} d T_{1} d l d x \\
=\int_{\alpha_{i}}^{q_{i}+\alpha_{i}} \int_{l_{a}}^{l_{b}}\left[\int_{T_{1 a}}^{T_{1 b}} T_{1} \frac{1}{\left(T_{1 b}-T_{1 a}\right)} d T_{1}\right] x^{-l} \frac{1}{\left(l_{b}-l_{a}\right)} d l d x \\
=\int_{\alpha_{i}}^{q_{i}+\alpha_{i}} \int_{l_{a}}^{l_{b}}\left[\frac{T_{1}^{2}}{2} \frac{1}{\left(T_{1 b}-T_{1 a}\right)}\right]_{T_{1} a}^{T_{1} b} x^{-l} \frac{1}{\left(l_{b}-l_{a}\right)} d l d x \\
=\int_{\alpha_{i}}^{q_{i}+\alpha_{i}} \frac{\left(T_{1 b}+T_{1 a}\right)}{2\left(l_{b}-l_{a}\right)} \int_{l_{a}}^{l_{b}} x^{-l} d l d x \\
x^{-l}=y \\
-\ln \ln (x)=\ln (y) \\
-\ln (x) d l=\frac{1}{y} d y \\
d l=\frac{1}{y} d y\left(\frac{-1}{\ln (x)}\right)
\end{gathered}
$$

The new limits are :

$$
\begin{gathered}
y_{a}=x^{-l_{a}} \\
y_{b}=x^{-l_{b}} \\
t^{s}\left(q_{i}\right)=\int_{\alpha_{i}}^{q_{i}+\alpha_{i}} \frac{\left(T_{1 b}+T_{1 a}\right)}{2\left(l_{b}-l_{a}\right)} \int_{y_{a}}^{y_{b}} y \frac{1}{y}\left(\frac{-1}{\ln (x)}\right) d y d x \\
=\int_{\alpha_{i}}^{q_{i}+\alpha_{i}} \frac{\left(T_{1 b}+T_{1 a}\right)}{2\left(l_{b}-l_{a}\right)}\left(-\frac{\left(x^{-l_{b}}-x^{-l_{a}}\right)}{\ln (x)}\right) d x
\end{gathered}
$$




\section{References}

Adler, G. and Nanda, R. (1974). The effects of learning on optimal lot size determinationsingle product case. AIIE Transactions, 6:14-20.

Amin, M. and Altiok, T. (1997). Control policies for multi-product multi-stage manufacturing systems: An experimental approach. International Journal of Production Research, 35:201-223.

Axsater, S. and Elmaghraby, S. (1981). A note on EMQ under learning and forgetting. AIIE Transactions, 13:86-90.

Bessant, J. and Haywood, B. (1988). Islands, archipelagoes and continents: Progress on the road to computer-integrated manufacturing. Research policy, 17:349-362.

Bokhorst, J., Slomp, J., and Molleman, E. (2004). Development and evaluation of crosstraining policies for manufacturing teams. IIE Transactions, 36:969-984.

Bollen, N. P. B. (1999). Real options and product life cycles. Management Science, $45: 670-684$.

Brusco, M. J. and Johns, T. R. (1998). Staffing a multiskilled workforce with varying levels of productivity: An analysis of cross-training policies. Decision Science, 29:499515.

Campbell, G. M. (1999). Cross-utilization of workers whose capabilities differ. Management Science, 45:722-732. 
Choi, S. and Kim, J. (1998). A study on the measurements of comprehensive flexibility in manufacturing systems. Computers in Industrial Engineering, 34:103-118.

Ebeling, A. and Lee, C. Y. (1994). Cross-training effectiveness and profitability. International Journal of Production Research, 32:2843-2859.

Epple, D., Argote, L., and Devadas, R. (1991). Organizational learning curves: A method for investigating intra-plant transfer of knowledge acquired through learning by doing. Organization Science, 2:58-70.

Gaury, E., Pierreval, H., and Kleijnen, J. (2000). An evolutionary approach to select a pull system among Kanban, CONWIP and hybrid. Journal of Intelligent Manufacturing, 11:157-167.

Geraghty, J. and Heavey, C. (2004). A comparison of hybrid push/pull and CONWIP/pull production inventory control policies. International Journal of Production Economics, 91:75-90.

Globerson, S., Levin, N., and Shtub, A. (1989). The impact of breaks on forgetting when performing a repetitive task. IIE Transactions, 21:376-381.

Gstettner, S. and Kuhn, H. (1996). Analysis of production control systems Kanban and CONWIP. International Journal of Production Research, 34:3253-3273.

Helber, S., Schimmelpfeng, K., and Stolletz, R. (2011). Setting inventory levels of CONWIP flow lines via linear programming. Official Open Access Journal of VHB, 4:98-115.

Herer, Y. and Masin, M. (1997). Mathematical programming formulation of CONWIP based production lines; and relationships to mrp. International Journal of Production Research, 35:1067-1076.

Hopp, W. and VanOyen, M. P. (2004). Agile workforce evaluation: A frame for crosstraining and coordination. IIE Transactions, 36:919-940. 
Hopp, W. J., Tekin, E., and VanOyen, M. P. (2004). Benefits of skill chaining in serial production lines with cross-trained workers. Management Science, 50:83-98.

Hull, J. C. (2002). Options, Futures, and Other Derivatives. Prentice Hall: New Jersey.

Hung, Y. and Chien, K. (2000). A multi-class multi-level capacitated lot sizing model. The Journal of the Operational Research Society, 51:1309-1318.

Iravani, S. M. R., Kolfal, B., and VanOyen, M. P. (2007). Call-center labor cross-training: It is small world after all. Management Science, 53:1102-1112.

Iravani, S. M. R., VanOyen, M. P., and Sims, K. T. (2005). Structural flexibility: A new perspective on the design of manufacturing and service operations. Management Science, 51:151-166.

Jaber, M. (2006). Lot sizing for an imperfect production process with quality corrective interruptions and improvements, and reduction in setups. Computers and Industrial Engineering, 51:781-790.

Jaber, M. and Bonney, M. (1996). Optimal lot sizing under learning considerations: The bounded learning case. Applied Mathematical Modelling, 20:750-755.

Jaber, M. and Bonney, M. (1997). The effects of learning and forgetting on the economic manufactured quantity (EMQ) with the consideration of intracycle backorders. International Journal of Production Economics, 53:1-11.

Jaber, M. and Bonney, M. (1998). The effects of learning and forgetting on the optimal lot size quantity of intermittent production runs. Production Planning and Control, 9:20-27.

Jaber, M. and Guiffrida, A. (2004). Learning curves for processes generating defects requiring reworks. European Journal of Operational Research, 159:663-672. 
Jaber, M. and Guiffrida, A. (2008). Learning curves for imperfect production processes with reworks and process restoration interruptions. European Journal of Operational Research, 189:93-104.

Jaber, M. and Kher, H. (2002). The dual-phased learning-forgetting model. International Journal of Production Economics, 76:229-242.

Jaber, M., Kher, H., and Davis, D. (2003). Countering forgetting through training and deployment. International Journal of Production Economics, 85:33-46.

Jaber, M. and Sikstrom, S. (2004). A numerical comparison of three potential learning and forgetting model. International Journal of Production Economics, 92:281-294.

Karwan, K., Mazzola, J., and Morey, R. (1988). Production lot sizing under setup and worker learning. Naval Research Logistics, 35:159-175.

Katok, E., Lewis, H., and Harrison, T. (1998). Lot sizing in general assemply systems with setup costs, setup times, and multiple constrained resources. Management Science, $44: 859-877$.

Marek, R. P., Elkins, D. A., and R.Smith, D. (2001). Understanding the fundamentals of Kanban and CONWIP pull systems using simulation. Proceedings of the 2001 Winter Simulation Conference, 1:921-929.

Marks, M. A., Sabella, M. J., Burke, C. S., and Zaccaro, S. J. (2002). The impact of cross-training on team effectiveness. Journal of Applied Psychology, 87:3-13.

Mazur, J. and Hastie, R. (1978). Learning as accumulation: A reexamination of the learning curve. Psychological Bulletin, 85:1256-1274.

Mazzola, J. and McCardle, K. (1997). The stochasitc learning curve: Optimal production in the presence of learning-curve uncertainty. Operations Research, 45:440-450. 
McCreery, J. and Krajewski, L. (1993). Improving performance using workforce flexibility in an assembly environment with learning and forgetting effects. International Journal of Production Research, 37:2031-2058.

Nembhard, D. (2001). A heuristic approach for assigning workers to tasks based on individual learning rate. International Journal of Production Research, 39:1955-1968.

Nembhard, D. and Shafer, S. (2008). The effects of workforce heterogeneity on productivity in an experiential learning environment. International Journal of Production Research, 46:3909-3929.

Nembhard, D. and Uzumeri, M. (2000). Experiential learning and forgetting for manual and cognitive tasks. International Journal of Industrial Ergonomics, 25:315-326.

Nembhard, D. A., Nembhard, H. B., and Qin, R. (2005). Real options model for workforce cross-training. The Engineering Economist, 50:95-116.

Nembhard, H., Nembhard, D., and Gurses, A. (2002). Real options modeling for valuing worker flexibility. Working paper, University of Wisconsin, Madison, WI.

Park, P. and Bobrowski, P. (1989). Job release and labor flexibility in a dual resource constrained job shop. Journal of Operations Management, 8:230-249.

Pratsini, E., Camm, J., and Raturi, A. (1993). Effect of process learning on manufacturing schedules. Computers and Operations Research, 20:15-24.

Ryan, S., Baynat, B., and Choobineh, F. (2000). Determining inventory levels in a CONWIP controlled job shop. IIE Transactions, 32:105-114.

Salameh, M., Abdul-Malak, M., and Jaber, M. (1993). Mathematical modeling of the effect of human learning in the finite production inventory model. Applied Mathematical Modeling, 17:613-615. 
Sethi, A. and Sethi, S. (1990). Flexibility in manufacturing: A survey. The International Journal of Flexible Manufacturing Systems, 2:289-328.

Shafer, S., Nembhard, D., and Uzumeri, M. (2001). The effects of worker learning, forgetting, and heterogeneity on assembly line productivity. Management Science, 47:1639-1653.

Shaw, D. and Wagelmans, A. (1998). An algorithm for single-item capacitated economic lot sizing with piecewise linear production costs and general holding costs. Management Science, 44:831-838.

Slomp, J. and Molleman, E. (2002). Cross-training policies and team performance. International Journal of Production Research, 40:1193-1219.

Smunt, T. (1987). The impact of worker forgetting on production scheduling. International Journal of Production Research, 25:689-701.

Spearman, M. L., Woodruff, D. L., and Hopp, W. J. (1990). CONWIP - a pull alternative to Kanban. International Journal of Production Research, 28:879-894.

Sule, D. (1981). Effect of learning and forgetting on economic lot size scheduling problem. International Journal of Production Research, 21:771-786.

Upton, D. (1994). The management of manufacturing flexibility. California Management Review, 36:72-89.

Upton, D. (1995). What really makes factories flexible? Harvard Business Review, $73: 74-84$.

VanMieghem, J. (1995). Investment strategies for flexible resources. Management Science, 44:1071-1078.

Wahab, M. and Lee, C. (2011). Pricing swing options with regime switching. Annals of Operations Research, 185:139-160. 
Wisner, J. and Siferd, S. (1995). A survey of US manufacturing practices in make-to-order machine shops. Production and Inventory Managament Journal, 36:1-6.

Wright, T. (1936). Factors affecting the cost of airplanes. Journal of Aeronautical Science, $3: 122-128$.

Yang, K. K. (2007). A comparison of cross-training policies in different job shops. International Journal of Production Research, 45:1279-1295.

Yelle, L. (1979). The learning curve: Historical review and comprehensive survey. Decision Sciences, 10:302-328.

Zamiska, J., Jaber, M., and Kher, H. (2007). Worker deployment in dual resource constrained systems with a task-type factor. European Journal of Operational Research, $177: 1507-1519$. 